\title{
Signature of the 2HDM at Higgs factories
}

\author{
Wenhai Xie $\odot,{ }^{1,2}$ R. Benbrik, ${ }^{3}$ Abdeljalil Habjia $\odot,{ }^{4}$ Souad Taj, ${ }^{4}$ Bin Gong, ${ }^{1}$ and Qi-Shu Yan ${ }^{2,5}$ \\ ${ }^{1}$ Theory Division, Institute of High Energy Physics, Chinese Academy of Sciences, Beijing 100049, China \\ ${ }^{2}$ School of Physics Sciences, University of Chinese Academy of Sciences, Beijing 100049, China \\ ${ }^{3}$ Laboratoire de Physique fondamentale et appliquée Safi, Faculté Polydisciplinaire de Safi, \\ Sidi Bouzid, B.P. 4162, Safi, Morocco \\ ${ }^{4}$ Sultan Moulay Slimane University, Polydisciplinary Faculty, Research Team in Theoretical Physics \\ and Materials (RTTPM), Beni Mellal 23000, Morocco \\ ${ }^{5}$ Center for Future High Energy Physics, Chinese Academy of Sciences, Beijing 100049, China
}

(Received 1 November 2019; accepted 14 April 2021; published 26 May 2021)

\begin{abstract}
The full one-loop corrections, both the weak and QED corrections, to the process $e^{+} e^{-} \rightarrow Z \phi$ $\left(\phi=h^{0}, H^{0}\right)$ in the two-Higgs-doublet model (2HDM) at the Higgs factories are presented. Up to the $O\left(\alpha_{\mathrm{em}}\right)$ level, the virtual corrections are evaluated by using the FeynArts/FormCalc packages. The real emission corrections are computed using the Feynman Diagram Calculation (FDC) package, and the collinear divergences are regularized by the electron structure functions. Using the FeynArts/FormCalc and FDC packages, we study the corrections in the Standard Model (SM) and the 2HDM, respectively. Gauge dependence arising in the renormalization of mixing angels is removed by using the pinch technique. After taking into account experimental constraints from the current LHC data, we propose four interesting benchmark scenarios for future colliders. By using these benchmark scenarios, we evaluate the deviation of $\Delta \sigma\left(e^{+} e^{-} \rightarrow Z \phi\right)$ from their SM values. We also examine Higgs boson decays $\phi \rightarrow b \bar{b}$ and $\phi \rightarrow \tau^{+} \tau^{-}$, which can have large electroweak (EW) contributions from triple Higgs couplings which are absent in the SM. It is found that for these benchmark scenarios, both EW and real emission corrections are sizeable and could be measured at future $e^{+} e^{-}$colliders such as the ILC, CLIC, and CEPC.
\end{abstract}

DOI: 10.1103/PhysRevD.103.095030

\section{INTRODUCTION}

A Higgs-like boson $(h)$ was discovered in the first run of the Large Hadron Collider (LHC) in 2012 [1,2]. Based on the data of Run 1, both the ATLAS and CMS Collaborations have established a Higgs boson mass with $m_{h}=125.09 \pm$ 0.21 (stat) \pm 0.11 (syst) GeV [3]. ATLAS and CMS have also performed several Higgs coupling measurements to a precision around $10 \%-20 \%$, such as Higgs couplings to dibosons $V V$ with $V=W^{ \pm}, Z, \gamma$ through a global fit with input from the measurement of Higgs decaying into two weak bosons, weak-vector-boson-associated processes, and vector boson fusion processes [4]. Very recently, ATLAS and CMS have also measured Higgs couplings to the fermions of the third generation over $5 \sigma$, via the process $p p \rightarrow V h(h \rightarrow b \bar{b})[5,6]$, the vector boson fusion process $p p \rightarrow j j h\left(h \rightarrow \tau^{+} \tau^{-}\right)[7,8]$, and $p p \rightarrow t t h$ with combined Higgs boson decay final states $[9,10]$. With Run 2 data, a

Published by the American Physical Society under the terms of the Creative Commons Attribution 4.0 International license. Further distribution of this work must maintain attribution to the author(s) and the published article's title, journal citation, and DOI. Funded by SCOAP. high precision for these measured Higgs boson couplings has been achieved $[11,12]$. These measurements demonstrate that the SM is consistent with the current Higgs data.

At the LHC, due to large theoretical uncertainties like the parton distribution function (PDF) and the large experimental background, the precision for the measured Higgs boson couplings can reach $4 \%-6 \%$ or so at $300 \mathrm{fb}^{-1}$ [13]. In contrast, due to its clean environment, an $e^{+} e^{-}$collider can perform precision measurements on the production and decay of the observed Higgs boson. For example, at a $240 \mathrm{GeV} e^{+} e^{-}$collider, the Higgs-strahlung process $e^{+} e^{-} \rightarrow Z h$ is the dominant Higgs production channel, where the Higgs boson can be reconstructed by using the recoiled $Z$ boson via its leptonic decay [14]. When a sufficiently large dataset is accumulated, say $1 \mathrm{ab}^{-1}$, the projected precision for the vertex $Z Z h$ can reach to $0.1 \%$ or so [15]. For a center-of-mass energy of $240-250 \mathrm{GeV}$ and an integrated luminosity of $250 \mathrm{fb}^{-1}$ for each of the two detectors in each year, a total $\mathcal{O}\left(10^{6}\right)$ Higgs bosons can be produced in a ten-year running (the total integrated luminosity can be estimated as $5 \mathrm{ab}^{-1}$ ), which can lead the precision of currently measured Higgs couplings to around one percent level or less [15-19]. At the International Linear Collider (ILC) experiments [20], the luminosity 
of a Higgs-factory run can be around $250 \mathrm{fb}^{-1}$ at $\sqrt{s}=250 \mathrm{GeV}$. The production rate of Higgs bosons from the Higgs-strahlung process can be enhanced when polarized beams [21] are used. However, at $\sqrt{s}=500 \mathrm{GeV}$ and/ or $\sqrt{s}=800 \mathrm{GeV}$, the vector-boson fusion process $W W \rightarrow$ $h$ can be dominant [21], and ILC will have the capability to reach a precision around $1 \%$ for most Higgs couplings [22]. Correspondingly, precise theoretical predictions of the physical observables related to Higgs bosons, like cross sections and branching ratios, are required.

In this paper, we propose some benchmark scenarios of the 2HDM after taking into account theoretical constraints as well as experimental restrictions from recent LHC data. By using these benchmark scenarios, we will study the effects of electroweak radiative corrections on the production process $e^{+} e^{-} \rightarrow Z \phi\left(\phi=h^{0}, H^{0}\right)$ and report full nextto-leading-order (NLO) calculations on the cross section in the 2HDM by including both virtual corrections up to one loop and real emissions of one photon. Our results are consistent with Ref. [23] within the same set of 2HDM parameters. The complete SM one-loop correction of $\sigma\left(e^{+} e^{-} \rightarrow Z h_{\mathrm{SM}}\right)$ was calculated for the first time in Ref. [24], then followed by Refs. [25,26]. Later on, it was calculated using the GRACE system ${ }^{1}$ in Ref. [28], and we reproduced the results for comparison. We find that the EW corrections of new physics in our benchmark scenarios can be of the order $-10 \% \sim-20 \%$, while the contribution of the real emission has a positive sign. Four benchmark points are studied in this paper and find that both EW and real emission corrections are sizeable, and could be the physical target at future $e^{+} e^{-}$colliders such as the ILC, CLIC, and CEPC.

One-loop corrections of the Higgs boson couplings have been investigated in various models. QCD and electroweak corrections of $h f \bar{f}$ were calculated in the SM in Refs. [2933]. Similar calculations have been done in various models beyond the SM, like in the minimal supersymmetric SM (MSSM). Due to their sizeable SUSY-QCD effects, it was observed that $h f \bar{f}$ couplings can receive large corrections [34-36]. Moreover, $h f \bar{f}$ couplings had been intensively studied in the 2HDM [37-39]. In this work, we also examine the deviations of $h f \bar{f}(f=b, \tau)$ in both type-I and type-II scenarios and find that these deviations are within the reach of future Higgs factories and are helpful in distinguishing new physics models.

The plan of the paper is as follows: In Sec. II, we introduce the benchmark points of the 2HDM model. In Sec. III, we outline the framework of our calculation and specify the renormalization scheme we will use. In Sec. IV, we present the numerical results. We conclude this work in Sec. V.

\footnotetext{
${ }^{1}$ GRACE is a system of program packages for automatic calculation; see [27].
}

\section{THE 2HDM AND BENCHMARK POINTS}

The 2HDM was first introduced by T. D. Lee [40] in order to have spontaneous T-parity breaking (or, equally, $C P$-parity breaking), and a more comprehensive review can be found in Refs. [41,42]. Before proposing benchmark scenarios, we briefly review the main features of the $2 \mathrm{HDM}$ model related to this work. ${ }^{2}$ The most general renormalizable potential which is invariant under $S U(2) \times U(1)$ can be written as

$$
\begin{aligned}
V\left(\Phi_{1}, \Phi_{2}\right)= & m_{1}^{2} \Phi_{1}^{\dagger} \Phi_{1}+m_{2}^{2} \Phi_{2}^{\dagger} \Phi_{2}-\left(m_{12}^{2} \Phi_{1}^{\dagger} \Phi_{2}+\text { H.c. }\right) \\
& +\frac{1}{2} \lambda_{1}\left(\Phi_{1}^{\dagger} \Phi_{1}\right)^{2}+\frac{1}{2} \lambda_{2}\left(\Phi_{2}^{\dagger} \Phi_{2}\right)^{2} \\
& +\lambda_{3}\left(\Phi_{1}^{\dagger} \Phi_{1}\right)\left(\Phi_{2}^{\dagger} \Phi_{2}\right)+\lambda_{4}\left(\Phi_{1}^{\dagger} \Phi_{2}\right)\left(\Phi_{2}^{\dagger} \Phi_{1}\right) \\
& +\left(\frac{1}{2} \lambda_{5}\left(\Phi_{1}^{\dagger} \Phi_{2}\right)^{2}+\left(\lambda_{6}\left(\Phi_{1}^{\dagger} \Phi_{1}\right)\right.\right. \\
& \left.\left.+\lambda_{7}\left(\Phi_{2}^{\dagger} \Phi_{2}\right)\right)\left(\Phi_{1}^{\dagger} \Phi_{2}\right)+\text { H.c. }\right),
\end{aligned}
$$

where $\Phi_{i}, i=1,2$ are complex $S U(2)$ doublets with four degrees of freedom each, and $m_{1}^{2}, m_{2}^{2}$, and $\lambda_{1-4}$ are all real, which follows from the Hermiticity of the potential. However, the parameters $\lambda_{5-7}$ and $m_{12}^{2}$ can be complex in general. Explicit $C P$ violation may arise in the Higgs sector by considering the imaginary parts of the above complex parameters. In this work, we focus on our study in the $C P$-conserving case, so we can assume all parameters to be real. Furthermore, for the sake of simplicity, we only include the soft breaking term proportional to $m_{12}^{2}$ and omit the terms proportional to $\lambda_{6}$ and $\lambda_{7}$, as these lead to hard $Z_{2}$ violation.

The $Z_{2}$ symmetry is defined as $\left(\Phi_{1}, \Phi_{2}\right) \rightarrow\left(\Phi_{1},-\Phi_{2}\right)$, which was introduced in order to suppress the tree-level flavor-changing neutral current (FCNC) processes [43,44]. Exact $Z_{2}$ symmetry will lead to the absence of $\Phi_{2}$ couplings into fermions, which makes $\Phi_{2}$ possible as a dark matter candidate in the so-called inert doublet model [45-47]. However, in this work, we consider the case $\lambda_{6}=\lambda_{7}=0$ but $m_{12}^{2} \neq 0$, which can allow FCNC processes at loop level $[43,48]$.

In the $C P$-conserving $2 \mathrm{HDM}$ case, four realizations have been considered in the $2 \mathrm{HDM}$ to avoid FCNC at the tree level, known as type I, type II, type III (called "leptonspecific") and type IV (also called "flipped") [49,50]. In the type-I model, the $Z_{2}$ symmetry is an exact symmetry, and the $\Phi_{1}$ doublet gives mass to all fermions. In the type-II model, the $\Phi_{1}$ doublet gives mass to leptons and down quarks, while the $\Phi_{2}$ couples to up-type quarks. The MSSM has a type-II Higgs sector. In the type-III model, all quarks couple to the $\Phi_{2}$ doublet, while all charged

\footnotetext{
${ }^{2}$ In this work, we follow the notations of Ref. [39], where more details can be found.
} 
TABLE I. Experimental results of the observables combined by the Particle Data Group (PDG) and/or Heavy Flavor Averaging Group (HFAG) Collaborations in Refs. [52-56]. As for $\overline{\mathcal{B}}\left(B_{q}^{0} \rightarrow \mu^{+} \mu^{-}\right.$), the combined results from the LHCb and CMS Collaborations are shown as given in Ref. [57]. Reference [58] is used for constraints from $(g-2)_{\mu}$ data.

\begin{tabular}{|c|c|c|c|}
\hline Observable & Experimental result & SM contribution & Combined error at $1 \sigma$ \\
\hline $\mathcal{B}(K \rightarrow \mu \nu) / \mathcal{B}(\pi \rightarrow \mu \nu)$ & $0.6357 \pm 0.0011[52]$ & $0.6231 \pm 0.0071$ & 0.0071 \\
\hline$\overline{\mathcal{B}}(b \rightarrow s \gamma)_{E_{\gamma}>1.6 \mathrm{GeV}}$ & $(3.32 \pm 0.16) \times 10^{-4}[55]$ & $(3.36 \pm 0.24) \times 10^{-4}$ & $0.29 \times 10^{-4}$ \\
\hline $\mathcal{B}(B \rightarrow \tau \nu)$ & $(1.14 \pm 0.22) \times 10^{-4}[56]$ & $(0.78 \pm 0.07) \times 10^{-4}$ & $0.23 \times 10^{-4}$ \\
\hline $\mathcal{B}(D \rightarrow \mu \nu)$ & $(3.74 \pm 0.17) \times 10^{-4}[52,56]$ & $(3.94 \pm 0.13) \times 10^{-4}$ & $0.21 \times 10^{-4}$ \\
\hline $\mathcal{B}\left(D_{s} \rightarrow \tau \nu\right)$ & $(5.55 \pm 0.24) \times 10^{-2}[52,56]$ & $(5.17 \pm 0.11) \times 10^{-2}$ & $0.26 \times 10^{-2}$ \\
\hline $\mathcal{B}\left(D_{s} \rightarrow \mu \nu\right)$ & $(5.57 \pm 0.24) \times 10^{-3}[52,56]$ & $(5.28 \pm 0.11) \times 10^{-3}$ & $0.26 \times 10^{-3}$ \\
\hline$\overline{\mathcal{B}}\left(B_{s}^{0} \rightarrow \mu^{+} \mu^{-}\right)$ & $(2.8 \pm 0.7) \times 10^{-9}[57]$ & $(3.66 \pm 0.28) \times 10^{-9}$ & $0.75 \times 10^{-9}$ \\
\hline$\overline{\mathcal{B}}\left(B_{d}^{0} \rightarrow \mu^{+} \mu^{-}\right)$ & $(3.9 \pm 1.5) \times 10^{-10}[57]$ & $(1.08 \pm 0.13) \times 10^{-10}$ & $1.50 \times 10^{-10}$ \\
\hline$\Delta M_{s}$ & $(17.757 \pm 0.021) \mathrm{ps}^{-1}[55]$ & $(18.257 \pm 1.505) \mathrm{ps}^{-1}$ & $1.5 \mathrm{ps}^{-1}$ \\
\hline$\Delta M_{d}$ & $(0.510 \pm 0.002) \mathrm{ps}^{-1}[55]$ & $(0.548 \pm 0.075) \mathrm{ps}^{-1}$ & $0.075 \mathrm{ps}^{-1}$ \\
\hline$\Delta_{0}\left(B \rightarrow K^{*} \gamma\right)$ & $(5.2 \pm 2.6) \times 10^{-2}[52]$ & $(5.1 \pm 1.5) \times 10^{-2}$ & $3.0 \times 10^{-2}$ \\
\hline$\delta a_{\mu}$ & $(261 \pm 80) \times 10^{-11}[58]$ & & $80 \times 10^{-11}$ \\
\hline
\end{tabular}

leptons couple to the $\Phi_{1}$ doublet. Finally, in the type-IV model, down-type quarks couple to the $\Phi_{1}$ doublet, while the rest of the fermions couple to the $\Phi_{2}$ doublet. With these four arrangements in the flavor space of 2HDM, the tree-level flavor-changing neutral current can be suppressed safely.

From the initial eight degrees of freedom, if the $S U(2)$ symmetry is broken, we end up with two $C P$-even Higgs states, usually denoted by $h^{0}$ and $H^{0}$; one $C P$-odd $A^{0}$; two charged Higgs bosons, $H^{ \pm}$; and three Goldstone bosons. After the electroweak symmetry breaking, the potential in Eq. (1) can be expressed with seven independent parameters: namely $m_{h^{0}}, m_{H^{0}}, m_{A^{0}}, m_{H^{ \pm}}, \tan \beta=v_{2} / v_{1}$, $\sin (\beta-\alpha)$, and $\lambda_{5}$ (or, equivalently, $m_{12}^{2}$ ). The angle $\beta$ is the rotation angle from the weak gauge eigenstates to the mass eigenstates in the $C P$-odd and charged Higgs sector. The angle $\alpha$ is the corresponding rotation angle for the $C P$-even sector. From this potential, the triple Higgs couplings needed for the present work can be derived, which are functions of the physical parameters and are given in Eqs. (B1)-(B8) of Appendix B.

The parameter space of the $2 \mathrm{HDM}$ is reduced by the following theoretical and experimental constraints:

1. Vacuum stability conditions that ensure the potential is bounded from below, where we use the conditions derived in Ref. [51].

2. Perturbative tree-level unitarity for scattering amplitudes of Higgs bosons and longitudinal components of gauge bosons.

3. The perturbativity of all quartic coefficients of the scalar potential: i.e., $\left|\lambda_{i}\right| \leq 8 \pi(i=1, \ldots, 5)$.

4. EW precision observables (EWPOs): Due to the contributions of extra Higgs bosons, the universal parameters $S, T$, and $U$ provide additional constraints on the mass splitting between these Higgs bosons. To implement the constraints from EWPOs, we consider the following values [52,53] for $S, T$, and $U: \Delta S=0.05 \pm 0.11, \quad \Delta T=0.09 \pm 0.13$, and $\Delta U=0.01 \pm 0.11$.

5. Indirect experimental constraints from $B$ physics observables, which have been taken into account by using the SuperIso [54] public code. Several important experimental values are tabulated in Table I, which will be used to constrain parameters such as $\tan \beta$ and the charged Higgs boson mass of the 2HDM significantly.

The 2HDMC public code [59] allows us to check all the listed constraints above. Furthermore, the proposed benchmark scenarios in this paper satisfy two more constraints: 1) the limits obtained from various searches for additional Higgs bosons at the LHC and other collider data, and 2) the requirement that there exist a neutral scalar which should match the measured properties of the Higgs-like boson. We evaluate the first constraint with the public code HiggsBounds5.3.2 [60-64], and the second constraint with the code HiggsSignals-2.2.3 [65-67].

Here we stress that after the Higgs-like particle discovery, several theoretical studies have performed global-fit analysis for the $2 \mathrm{HDM}$ to pinpoint the allowed regions of parameter space for a SM-like Higgs $h^{0}$ [68-75], as well as for a SM-like Higgs boson $H^{0}[76,77]$. Before presenting our results, we would like to mention that we have performed a cross-check of the results in Ref. [23] for the subclass of Yukawa corrections considered there and found perfect agreement.

To explore the effect of new physics in the 2HDM in our following study, we propose four benchmark points (BPs), as shown in Table II, based on the best fit from the latest results of Higgs data using the HiggsBounds and HiggsSignals public codes. 
TABLE II. Selected benchmark points using Higgs data at $13 \mathrm{TeV}$ with $m_{h}=125 \mathrm{GeV}$ are presented for BP1-h and BP2-h, and with $m_{H}=125 \mathrm{GeV}$ for BP1-H and BP2-H. Obviously, in this notation, the BP1 (BP2) type is related to type-I (type-II) 2 HDM. Here we adopt four physical masses, two angles, and $\lambda_{5}$ to describe each point in the parameter space. In each BP, we examine the ratio of total decay width over the mass of a Higgs boson-i.e., $\Gamma_{\phi} / m_{\phi}$-and find that it is smaller than $5 \%$ with $\phi=H, A$ and $H^{ \pm}$.

\begin{tabular}{lrrrrrrr}
\hline \hline BPs & $\sin (\beta-\alpha)$ & \multicolumn{1}{c}{$\tan \beta$} & $m_{h}(\mathrm{GeV})$ & $m_{H}(\mathrm{GeV})$ & $m_{A}(\mathrm{GeV})$ & $m_{H^{ \pm}}(\mathrm{GeV})$ & \multicolumn{1}{c}{$\lambda_{5}$} \\
\hline BP1-h & 0.99679 & 14.300 & 125.00 & 212.00 & 98.20 & 178.27 & 0.5819 \\
BP2-h & 0.99999 & 2.012 & 125.00 & 594.00 & 512.00 & 592.00 & 0.0000 \\
BP1-H & -0.06000 & 2.830 & 95.00 & 125.00 & 169.00 & 170.00 & -0.3220 \\
BP2-H & -0.03000 & 2.160 & 95.00 & 125.00 & 600.00 & 600.00 & -5.7800 \\
\hline \hline
\end{tabular}

To demonstrate the impact of experimental data as well as theoretical constraints upon the parameter spaces, we perform a scan on $\sin (\beta-\alpha) / \cos (\beta-\alpha)$ and the $\tan \beta$ plane. The results are shown in Fig. 1, where we project the LHC constraints discussed above onto allowed regions at a 95\% confidence level (C.L.) (in yellow), as well as at a $68 \%$ C.L. (in green) in the $[\sin (\beta-\alpha), \tan \beta]$ plane for $h$ being SM-like for type-I (upper-left panel) and type-II (upper-right panel) 2HDM. In the lower plots, the heavier Higgs boson $H$ is the SM-like Higgs boson with the same
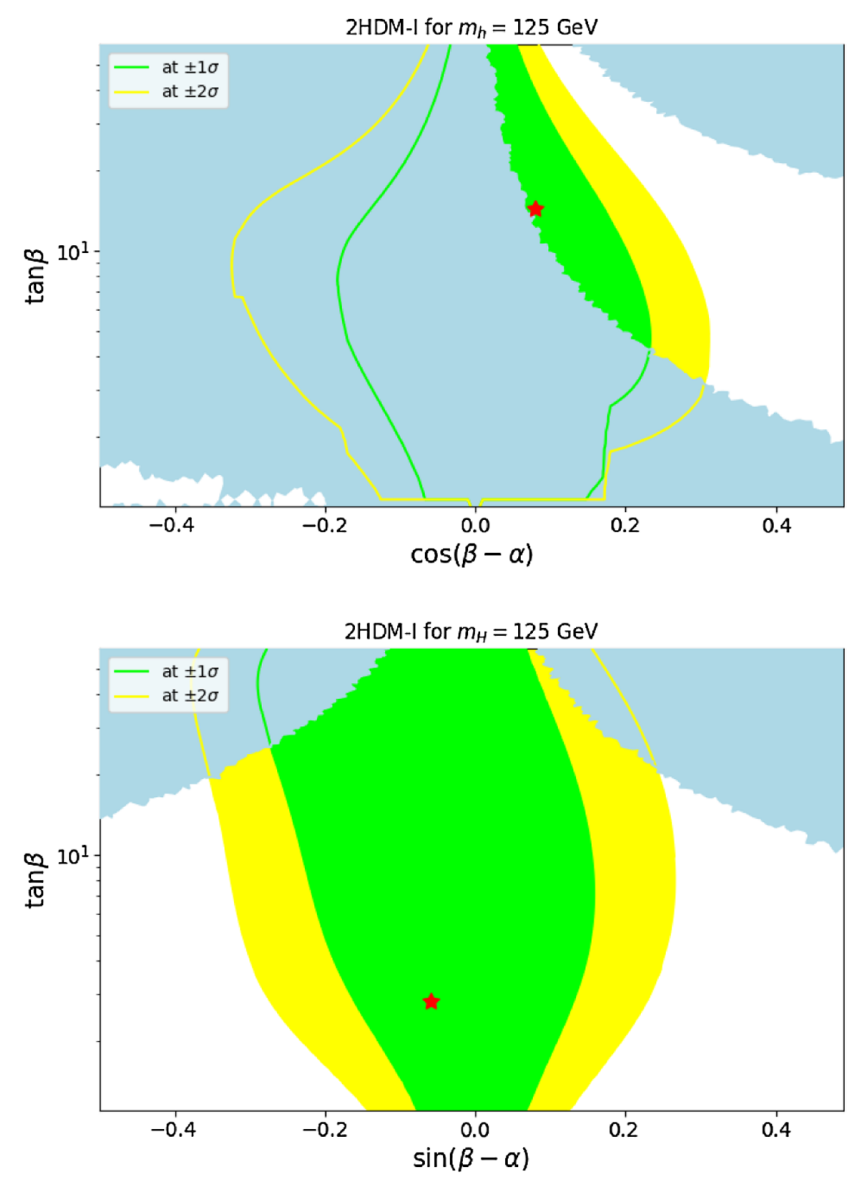

coding color. The four selected benchmark points are given in Table II, each of which corresponds to a red star in the plots of Fig. 1.

Concerning the mass of the charged Higgs boson, here we would like to point out that a light charged Higgs boson less than $200 \mathrm{GeV}$ can be consistent with the current LHC Higgs data. As is well known, in the framework of 2HDMII and -IV, for example, the measurement of the $b \rightarrow s \gamma$ branching ratio requires the mass of the charged Higgs boson to be heavier than $580 \mathrm{GeV}[78,79]$ for any value of
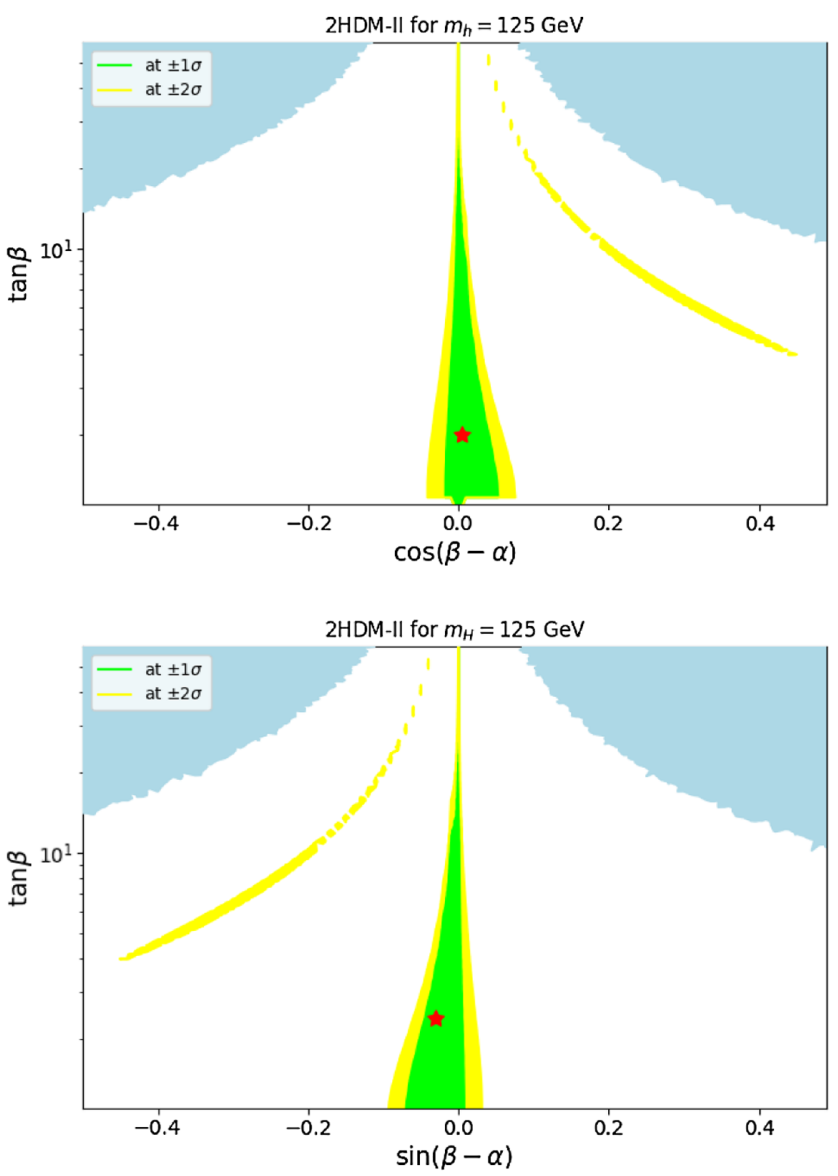

FIG. 1. Allowed ranges at 95\% C.L. (yellow) and 68\% C.L. (green) from direct search data in LHC Run 2 are shown. Regions excluded by theoretical constraints are shown in cyan. A red star corresponds to the best-fit point for each scenario. 


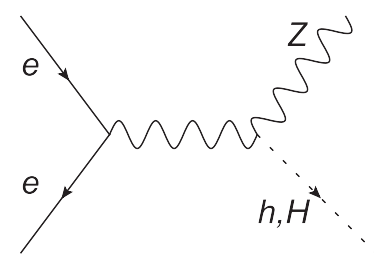

FIG. 2. The LO Feynman diagram for the processes $e^{+} e^{-} \rightarrow$ $Z h$ and $e^{+} e^{-} \rightarrow Z H$ in the 2HDM is shown.

$\tan \beta \geq 1$. Such a limit is much lower for the other 2HDM types [80]. In 2HDM-I and -III, as long as $\tan \beta \geq 2$, there are allowed regions in the parameter space with a charged Higgs boson as light as $100 \mathrm{GeV}[80,81]$ that are still consistent with all $B$ physics constraints, as well as with LEP and LHC limits, as shown in the literature [82-87].

Due to the tiny Yukawa coupling of the Higgs/Goldstone boson to the electron, the overwhelming and leading-order (LO) contribution for the processes $e^{+} e^{-} \rightarrow Z h$ and $e^{+} e^{-} \rightarrow Z H$ in the 2HDM comes from the Feynman diagram given in Fig. 2. Then the total tree-level cross section in the 2HDM, $\sigma^{0}$, can be expressed as

$$
\begin{aligned}
\sigma^{0}\left(e^{+} e^{-} \rightarrow Z \phi\right)= & \sin ^{2}(\beta-\alpha)\left[\cos ^{2}(\beta-\alpha)\right] \\
& \times \sigma_{\mathrm{SM}}^{0}\left(e^{+} e^{-} \rightarrow Z h\right) \text { for } \phi=h[H],
\end{aligned}
$$

where the tree-level cross section of the SM $\sigma_{\mathrm{SM}}^{0}$ is defined as

$$
\sigma_{\mathrm{SM}}^{0}\left(e^{+} e^{-} \rightarrow \mathrm{Zh}\right)=\frac{\alpha_{\mathrm{em}}^{2} \pi}{192 s s_{W}^{4} c_{W}^{4}}\left(v_{e}^{2}+a_{e}^{2}\right) \lambda^{\frac{1}{2}} \frac{\lambda+12 m_{\mathrm{Z}}^{2} / s}{\left(1-m_{\mathrm{Z}}^{2} / s\right)^{2}},
$$

where $s$ is the squared collision energy, $a_{e}=-1, v_{e}=$ $-1+4 \sin ^{2} \theta_{W}, \quad \alpha_{\mathrm{em}}=e^{2} / 4 \pi, \quad \lambda=\left(1-m_{h}^{2} / s-m_{Z}^{2} / s\right)^{2}-$ $4 m_{h}^{2} m_{Z}^{2} / s^{2}$. Here we have used $s_{W}$ and $c_{W}$ for $\sin \left(\theta_{W}\right)$ and $\cos \left(\theta_{W}\right)$, and $\theta_{W}$ is the Weinberg angle. With the relation $\alpha_{\mathrm{em}}=\sqrt{2} m_{W}^{2} s_{W}^{2} G_{F} / \pi$, it can be seen that our LO result in the SM is consistent with those in Refs. [88-90]. The formula of tree-level cross sections for Higgs-strahlung processes at $e^{+} e^{-}$colliders in the 2HDM can be also found in Ref. [91], where the MSSM was considered, which has a built-in type-II $2 \mathrm{HDM}$ in the Higgs sector.

\section{ONE-LOOP RENORMALIZATION AND CALCULATION}

Calculations of higher-order corrections in perturbation theory in general lead to ultraviolet (UV) divergences. The standard procedure to eliminate these UV divergences consists in the renormalization of the bare Lagrangian by redefining the couplings and fields. In the SM, the on-shell renormalization scheme is well elaborated [92-94].
As shown in Ref. [94], the renormalization constant for charge $\delta Z_{e}$ is obtained as

$\delta Z_{e}=-\frac{1}{2} \delta Z_{A A}-\frac{s_{W}}{c_{W}} \frac{1}{2} \delta Z_{Z A}=\frac{1}{2} \Pi(0)-\frac{s_{W}}{c_{W}} \frac{\sum_{T}^{A Z}(0)}{m_{Z}^{2}}$,

with

$$
\Pi(s) \equiv \frac{\sum_{T}^{A A}(s)}{s}
$$

and

$$
\Pi(0)=\lim _{s \rightarrow 0} \frac{\sum_{T}^{A A}(s)}{s}=\left.\frac{\partial \sum_{T}^{A A}(s)}{\partial s}\right|_{s=0},
$$

where the definitions for $\delta Z_{e}, \delta Z_{A A}$ and $\delta Z_{Z A}$ can be found in Eqs. (C2) and (C3). This corresponds to the running coupling constant obtained in the Thomson limit, taken as $\alpha_{\mathrm{em}}\left(m_{e}\right)$ or $\alpha_{\mathrm{em}}(0)$. The vacuum polarization $\Pi(0)$, the first term in $\delta Z_{e}$, is sensitive to the hadronic contribution. Usually, a nonperturbative parameter $\Delta \alpha_{\text {hadron }}^{(5)}\left(m_{Z}\right)$ (" 5 " here is the number of quark flavors, which means the top quark is not included) is used to absorb the hadronic contribution; i.e., $\Pi(0)$ is modified as (the contributions from leptons are also separated for further discussion)

$$
\begin{aligned}
\Pi(0)= & \Pi_{\text {hadron }}^{(5)}(0)+\Pi_{\text {lepton }}(0)+\Pi_{\text {remaining }}(0) \\
\rightarrow & \operatorname{Re} \Pi_{\text {hadron }}^{(5)}\left(m_{Z}^{2}\right)+\Delta \alpha_{\text {hadron }}^{(5)}\left(m_{Z}\right) \\
& +\Pi_{\text {lepton }}(0)+\Pi_{\text {remaining }}(0)
\end{aligned}
$$

with $\Delta \alpha_{\text {hadron }}^{(5)}\left(m_{Z}\right)=0.02764$ according to PDG data [95]. Then, $\delta Z_{e}$ is rewritten as

$$
\begin{aligned}
\delta Z_{e}(0)= & \frac{1}{2} \operatorname{Re} \Pi_{\text {hadron }}^{(5)}\left(m_{Z}^{2}\right)+\frac{1}{2} \Delta \alpha_{\text {hadron }}^{(5)}\left(m_{Z}\right)+\frac{1}{2} \Pi_{\text {lepton }}(0) \\
& +\frac{1}{2} \Pi_{\text {remaining }}(0)-\frac{s_{W}}{c_{W}} \frac{\sum_{T}^{A Z}(0)}{m_{Z}^{2}}
\end{aligned}
$$

In the following text and Table III, we label the scheme defined in Eq. (8) as the $\alpha_{\mathrm{em}}(0)$ scheme. Meanwhile, whenever $\Pi(0)$ is mentioned, it refers to the one defined in Eq. (7).

Two additional schemes, labeled as $\alpha_{\mathrm{em}}\left(m_{Z}\right)$ and $\alpha_{\mathrm{em}}(\sqrt{s})$, are defined below, where the large logarithmic contributions of leptons are also absorbed into the redefinition of the running coupling constant $[94,96]$ : 


$$
\begin{aligned}
\delta Z_{e}(\mu) \equiv & \delta Z_{e}(0)-\frac{1}{2} \Delta \alpha(\mu)=\frac{1}{2} \operatorname{Re} \Pi_{\text {hadron }}^{(5)}\left(\mu^{2}\right) \\
& +\frac{1}{2} \operatorname{Re} \Pi_{\text {lepton }}\left(\mu^{2}\right)+\frac{1}{2} \Pi_{\text {remaining }}(0)-\frac{s_{W}}{c_{W}} \frac{\sum_{T}^{A Z}(0)}{m_{Z}^{2}},
\end{aligned}
$$

with

$$
\begin{aligned}
\Delta \alpha(\mu) \equiv & \Pi_{f \neq \text { top }}(0)-\operatorname{Re} \Pi_{f \neq \text { top }}\left(\mu^{2}\right) \\
= & {\left[\operatorname{Re} \Pi_{\text {hadron }}^{(5)}\left(m_{Z}^{2}\right)+\Delta \alpha_{\text {hadron }}^{(5)}\left(m_{Z}\right)-\operatorname{Re} \Pi_{\text {hadron }}^{(5)}\left(\mu^{2}\right)\right] } \\
& +\left[\Pi_{\text {lepton }}(0)-\operatorname{Re} \Pi_{\text {lepton }}\left(\mu^{2}\right)\right] .
\end{aligned}
$$

And the running coupling constant is defined as

$$
\alpha_{\mathrm{em}}(\mu) \equiv \frac{\alpha_{\mathrm{em}}(0)}{1-\Delta \alpha(\mu)} .
$$

In the $\alpha_{\mathrm{em}}\left(m_{Z}\right)$ scheme, we take $\mu=m_{Z}$, while in the $\alpha_{\mathrm{em}}(\sqrt{s})$ scheme, we take $\mu=\sqrt{s}$. Results in these two schemes will be independent of $\log \left(m_{e}\right)$. In the following, we use the $\alpha_{\mathrm{em}}\left(m_{Z}\right)$ scheme as our default choice. In the procedure of renormalization using dimensional regularization, a scale $\mu_{r}$ is introduced. Usually this will generate a $\operatorname{logarithmic}$ term, $\log \left(\mu_{r}\right)$. As we use a complete on-shell scheme in both production processes, such terms which can be considered as an overall factor of $\left(\mu_{r}^{2}\right)^{\epsilon}$ in each individual part [counterterms in the modified minimal subtraction $(\overline{\mathrm{MS}})$ scheme do not have such a factor] will vanish upon the cancellation of UV and infrared (IR) divergences when summing over all the parts. This leads to $\mu_{r}$ independence of our results for the production processes. On the other hand, from Eq. (9) a new scale $\mu$ is introduced that denotes the scale at which charge is renormalized, and our results are dependent on it. From now on, when we talk about renormalization scale, it denotes $\mu$, which is introduced in the renormalization of charge, not $\mu_{r}$.
The renormalization of $2 \mathrm{HDM}$ had been plagued by the issue of the gauge dependence of the mixing parameters, like $\tan \beta$, as in the MSSM case where type-II 2HDM is needed [97-99]. A reasonable and convenient scheme should be gauge independent, process independent, and numerically stable [100]. Based on the renormalization scheme worked out by Fleischer and Jegerlehner in Ref. [32], which is now usually called the FJ tadpole scheme, two groups find a way to fulfill such conditions. The first one is the $\overline{\mathrm{MS}}$ tadpole scheme (MSTS) [101-103], where the mixing angles are renormalized using $\overline{\mathrm{MS}}$ subtraction. The other is the pinched tadpole scheme (PTS) $[104,105]$, where the pinch technique (see, e.g., Ref. [106]) is used to define gauge-independent counterterms for the mixing angles. More renormalization schemes have been proposed and examined numerically [107,108] and have been implemented in the HDecay package [109].

In this work, we adopt the on-shell PTS described in Ref. [104]. "On-shell" here means that the on-shell scale is chosen in the self-energies during the renormalization of the mixing angles. More details about the renormalization scheme in this work can be found in Appendix C.

In our calculation, a small photon mass $\lambda$ is introduced to regularize the soft divergence. Meanwhile, two cutoffs, $\Delta E$ and $\Delta \theta$, are introduced to deal with the IR singularities in real correction processes. The three-body phase space of the real correction process $e^{+} e^{-} \rightarrow Z h \gamma$ is divided into three parts:

1. Soft $(S)$ part: The energy of photon $E_{\gamma}$ is smaller than $\Delta E$.

2. Hard collinear (HC) part: $E_{\gamma} \geq \Delta E$, and the angle between the photon and the beam $\theta_{\gamma}$ is smaller than $\Delta \theta$.

3. Hard noncollinear $(H \bar{C})$ part: The remaining, which is finite.

Thus, next-to-leading-order (NLO) corrections can be expressed as

$$
d \sigma^{1}=d \sigma_{V}(\lambda)+d \sigma_{S}(\lambda, \Delta E)+d \sigma_{\mathrm{HC}+\mathrm{CT}}(\Delta E, \Delta \theta)+d \sigma_{H \bar{C}}(\Delta E, \Delta \theta) .
$$

Here, $d \sigma_{V}$ denotes the virtual correction, including loop diagrams and counterterms from renormalization.

The soft part is given by

$$
d \sigma_{S}=-\frac{\alpha_{\mathrm{em}}}{\pi} d \sigma^{0} \times\left[\log \frac{4 \Delta E^{2}}{\lambda^{2}}\left(1+\log \frac{m_{e}^{2}}{s}\right)+\frac{1}{2} \log ^{2} \frac{m_{e}^{2}}{s}+\log \frac{m_{e}^{2}}{s}+\frac{1}{3} \pi^{2}\right],
$$

where $d \sigma^{0}$ denotes the tree-level differential cross section. The hard collinear part is obtained in the collinear limit as

$$
\begin{aligned}
d \sigma_{\mathrm{HC}} & =\frac{\alpha_{\mathrm{em}}}{2 \pi}\left[\frac{1+z^{2}}{1-z} \log \frac{\Delta \theta^{2}+4 m_{e}^{2} / s}{4 m_{e}^{2} / s}-\frac{2 z}{1-z} \frac{\Delta \theta^{2}}{\Delta \theta^{2}+4 m_{e}^{2} / s}\right] d \sigma^{0}\left(z k_{1}\right) d z+\left(k_{1} \Leftrightarrow k_{2}\right) \\
& \stackrel{\Delta \theta^{2} \gg m_{e}^{2} / s}{\longrightarrow} \frac{\alpha_{\mathrm{em}}}{2 \pi}\left[\frac{1+z^{2}}{1-z} \log \frac{\Delta \theta^{2} s}{4 m_{e}^{2}}-\frac{2 z}{1-z}\right] \times\left[d \sigma^{0}\left(z k_{1}\right)+d \sigma^{0}\left(z k_{2}\right)\right] d z,
\end{aligned}
$$


with $0 \leq z \leq 1-\delta_{s}=1-2 \Delta E / \sqrt{s}$, which is also related to the tree-level differential cross section $d \sigma^{0}$. CT denotes the "counterterm" from the electron structure function, which originates from the second term in Eq. (A1):

$$
d \sigma_{\mathrm{CT}}=-\frac{\alpha_{\mathrm{em}}}{2 \pi} \log \frac{s}{4 m_{e}^{2}} P_{e e}^{+}(z, 0) \times\left[d \sigma^{0}\left(z k_{1}\right)+d \sigma^{0}\left(z k_{2}\right)\right] d z,
$$

with $0 \leq z \leq 1$. Thus, the combination of $\mathrm{HC}$ and $\mathrm{CT}$ parts can be expressed as

$$
\begin{aligned}
d \sigma_{\mathrm{HC}+\mathrm{CT}} & \equiv d \sigma_{\mathrm{HC}+\mathrm{CT}}^{*}+d \sigma_{\mathrm{SC}} \\
d \sigma_{\mathrm{HC}+\mathrm{CT}}^{*} & =\frac{\alpha_{\mathrm{em}}}{2 \pi}\left[\frac{1+z^{2}}{1-z} \log \Delta \theta^{2}-\frac{2 z}{1-z}\right] \times\left[d \sigma^{0}\left(z k_{1}\right)+d \sigma^{0}\left(z k_{2}\right)\right] d z \\
d \sigma_{\mathrm{SC}} & =-\frac{\alpha_{\mathrm{em}}}{\pi} \log \frac{s}{4 m_{e}^{2}}\left[\frac{3}{2}+2 \log \delta_{s}\right] d \sigma^{0} .
\end{aligned}
$$

Both the soft and virtual parts are obtained with FormCalc, while the other parts are obtained with the help of FDC [110]. The $\lambda$ dependence has been checked when we combine the soft and virtual parts; more detailed checking in the SM can be found in Appendix A.

In Appendix A, we examine the dependence of the SM cross section on those unphysical parameters, such as $\delta_{s}$ in Table IV and $\Delta \theta$ in Table V. From these results, it is observed that within a reasonable region where $\delta_{s} \ll 1$ and $m_{e} / \sqrt{s} \ll \Delta \theta \ll 1$ are satisfied, our results are indeed independent of those unphysical parameters introduced by the algorithm. Meanwhile, as we adopt the $\alpha_{\mathrm{em}}\left(m_{Z}\right)$ scheme, the cross section should also be independent of the logarithm term of the electron mass, $\log \left(m_{e}\right)$. This independence is shown in Table VI. After these checks, we apply our procedure to the $2 \mathrm{HDM}$; i.e., we calculate the one-loop radiative corrections to the Higgs-strahlung process $e^{+} e^{-} \rightarrow Z \phi$.

Here we calculate the radiative corrections to the treelevel $e^{+} e^{-} \rightarrow Z h^{0}, Z H^{0}$ processes in 2HDM in the Feynman-'t Hooft gauge, including all the particles of the model in the loops. Counterterms are constructed by specific renormalization conditions which allow us to cancel all the UV divergences with one-loop diagrams. Inserting these redefinitions into the Lagrangian, we find the following counterterms for $h^{0} \mathrm{ZZ}$ and $H^{0} \mathrm{ZZ}$ :

$$
\begin{aligned}
\delta \mathcal{L}_{h^{0} Z Z}= & \frac{e m_{W} \sin (\beta-\alpha)}{s_{W} c_{W}^{2}}\left[\delta Z_{e}-\frac{\delta s_{W}}{s_{W}}-\frac{2 \delta c_{W}}{c_{W}}+\frac{\delta m_{W}^{2}}{2 m_{W}^{2}}+\frac{1}{2} \delta Z_{h^{0} h^{0}}+\delta Z_{Z Z}\right. \\
& \left.+\frac{1}{2} \cot (\beta-\alpha) \delta Z_{H^{0} h^{0}}+\cot (\beta-\alpha)(\delta \beta-\delta \alpha)\right] h^{0} Z^{\mu} Z^{\nu} g_{\mu \nu} \\
\delta \mathcal{L}_{H^{0} Z Z}= & \frac{e m_{W} \cos (\beta-\alpha)}{s_{W} c_{W}^{2}}\left[\delta Z_{e}-\frac{\delta s_{W}}{s_{W}}-\frac{2 \delta c_{W}}{c_{W}}+\frac{\delta m_{W}^{2}}{2 m_{W}^{2}}+\frac{1}{2} \delta Z_{H^{0} H^{0}}+\delta Z_{Z Z}\right. \\
& \left.+\frac{1}{2} \tan (\beta-\alpha) \delta Z_{h^{0} H^{0}}-\tan (\beta-\alpha)(\delta \beta-\delta \alpha)\right] H^{0} Z^{\mu} Z^{\nu} g_{\mu \nu} .
\end{aligned}
$$

The one-loop Feynman diagrams related to the processes $e^{+} e^{-} \rightarrow Z \phi_{i}$ are displayed in Figs. 3-6, which are conveniently dubbed as the vertex corrections, the box contributions, the self-energy corrections, and the counterterms, respectively. In these Feynman diagrams, the labels $V, S$, and $F$ denote all insertions of vector, scalar, and fermionic states of the 2HDM. As we used the onshell PTS in our renormalization scheme, we should also include all possible tadpole diagrams, which have been merged to corresponding counterterm diagrams in Fig. 6 and have not been shown explicitly.
There is one issue concerning the effects of the widths of particles in the $s$-channel diagrams which we need to clarify. For the processes that we consider here, the intermediate $Z$ boson is always far away from its mass shell, as the collision energy is assumed to be $250 \mathrm{GeV}$ or even higher. Thus, it is plausible to neglect the effect of the width of the $Z$ boson in our calculation. Meanwhile, it is observed that contributions from initial-state $e^{+} e^{-} \phi$ vertices vanish in the limit $m_{e} \rightarrow 0$ since both $e^{+}$and $e^{-}$ are on shell, so it is justifiable to neglect the widths of scalar bosons. Obviously, such an argument holds for 


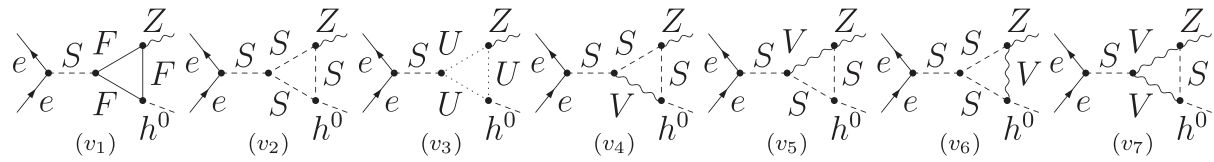

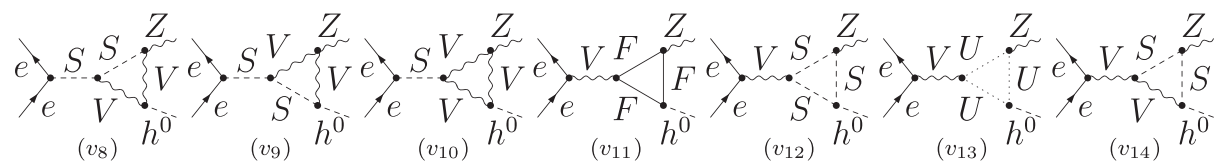

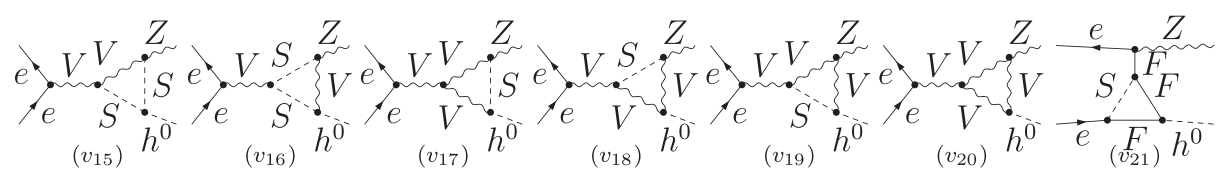

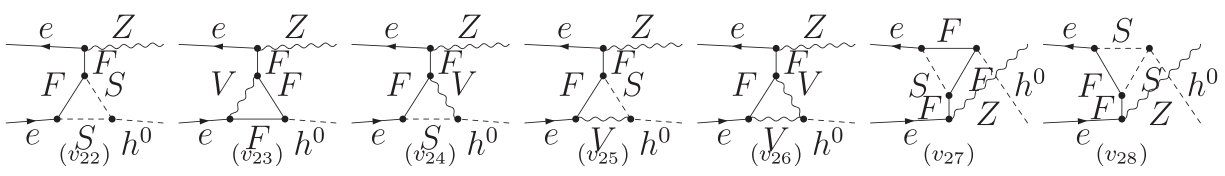

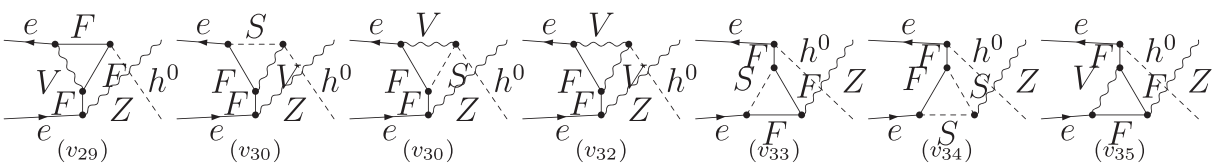

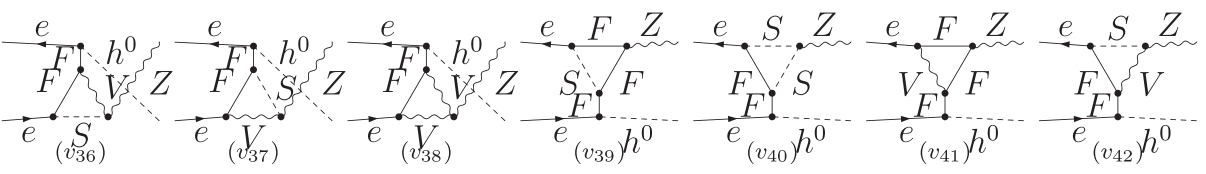

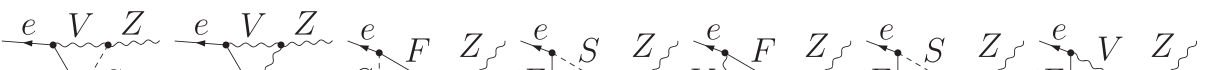

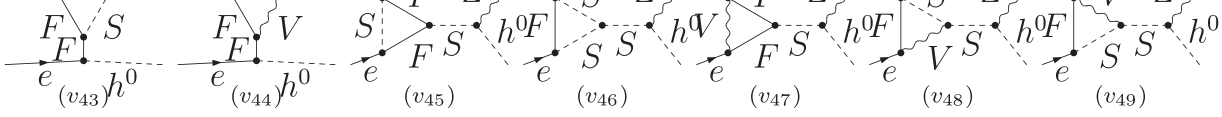

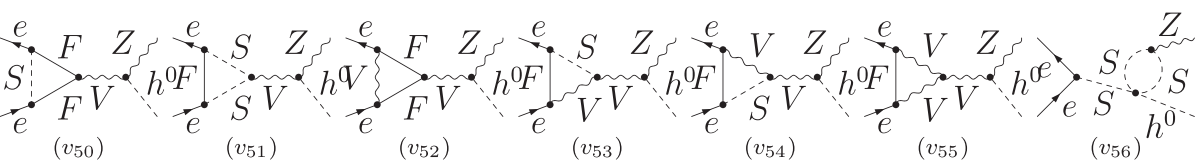

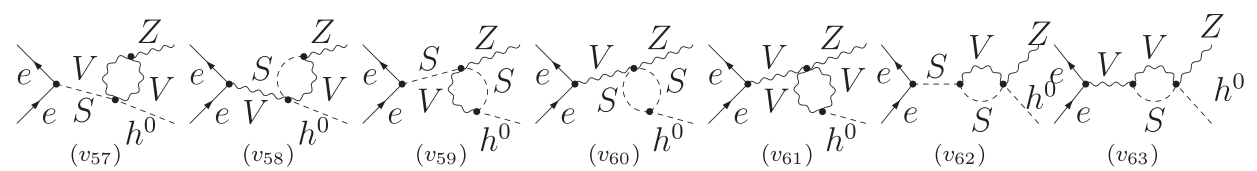

FIG. 3. Generic three-point one-loop Feynman diagrams in the 2HDM contributing to the process $e^{+} e^{-} \rightarrow Z h^{0}\left(\right.$ we take $\left.\phi=h^{0}\right)$ are presented, where the labels $U, V, S$, and $F$ refer to ghosts, vector gauge bosons, Higgs scalar bosons, and fermions, respectively.

the rest of the scalar boson exchange diagrams in the $s$ channel. As mentioned in the caption of Table II, the ratio $\Gamma / m$ for all the new Higgs boson particles is less than $5 \%$, and the narrow-width approximation can hold. Therefore, in this work, for those $s$-channel diagrams, we simply neglect the widths of the $Z$ boson and other scalar bosons.

Finally, the total cross section at NLO, denoted as $\sigma^{\mathrm{NLO}}$, is defined as the sum of the LO cross section, $\sigma^{0}$, and the NLO corrections, $\sigma^{1}$-i.e.,

$$
\sigma^{\mathrm{NLO}}=\sigma^{0}+\sigma^{1} \equiv \sigma^{0}\left(1+\delta^{1}\right),
$$

where $\delta^{1}$ is defined as the ratio which measures the relative strength of next-to-leading-order corrections over the treelevel result.
As described in Sec. 3.1 of Ref. [111], the NLO electroweak corrections $\sigma^{1}$ can be safely grouped into two gauge-invariant parts:

1. The "QED" part, which includes all the diagrams which contain an extra photon attached to the LO diagrams, such as the diagrams $v_{23}, v_{29}$, and $v_{52}$ in Fig. 3 when the vector boson denotes a photon. Meanwhile, the photon's contribution to the wavefunction renormalization of the electron is also grouped into this part.

2. The "weak" part, which contains all the remaining contributions.

In terms of the convention introduced in Ref. [111], we can divide $\sigma^{1}$ as 


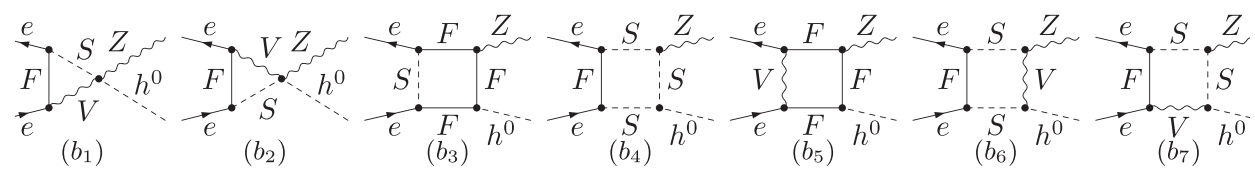

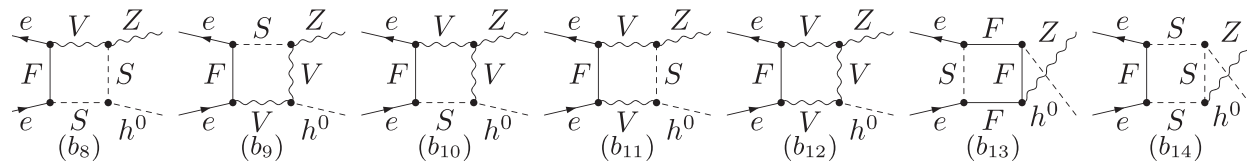

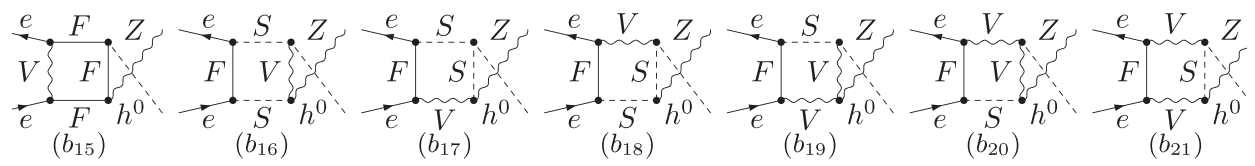

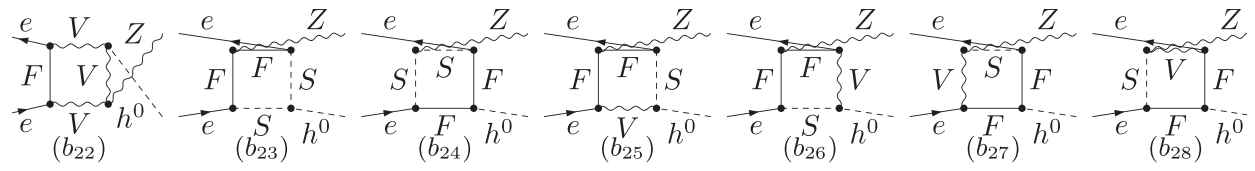

$$
\begin{aligned}
& \text { P. }
\end{aligned}
$$

FIG. 4. Generic four-point one-loop Feynman diagrams in the 2HDM contributing to the process $e^{+} e^{-} \rightarrow Z h^{0}$ (we take $\phi=h^{0}$ ) are shown, where the labels of $V, S$, and $F$ refer to vector gauge bosons, Higgs scalar bosons, and fermions, respectively.

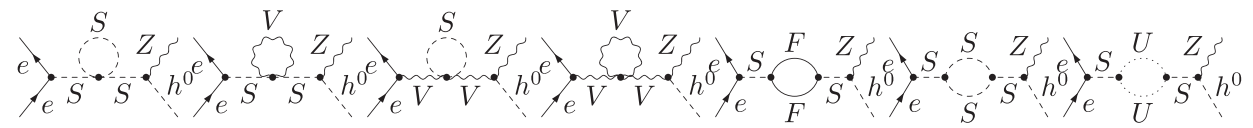

$$
\begin{aligned}
& \begin{array}{lllllll}
\left(s_{1}\right) & \left(s_{2}\right) & \left(s_{3}\right) & \left(s_{4}\right) & \left(s_{5}\right) & \left(s_{6}\right) & \left(s_{7}\right)
\end{array}
\end{aligned}
$$

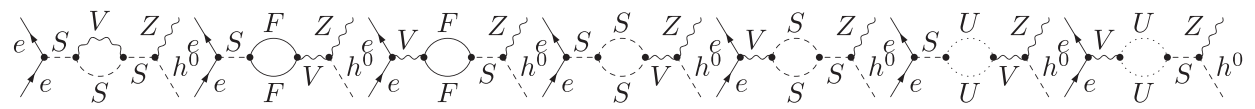

$$
\begin{aligned}
& \begin{array}{lllllll}
\left(s_{8}\right) & \left(s_{9}\right) & \left(s_{10}\right) & \left(s_{11}\right) & \left(s_{12}\right) & \left(s_{13}\right) & \left(s_{14}\right)
\end{array}
\end{aligned}
$$

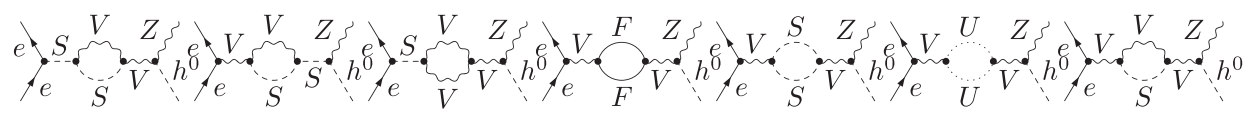

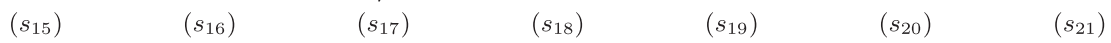

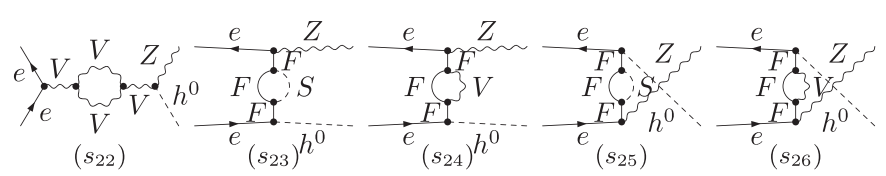

FIG. 5. Generic two-point one-loop Feynman diagrams in the 2HDM contributing to the process $e^{+} e^{-} \rightarrow Z h^{0}$ (we take $\phi=h^{0}$ ) are shown, where the labels of $U, V, S$, and $F$ refer to ghosts, vector gauge bosons, Higgs scalar bosons, and fermions, respectively.

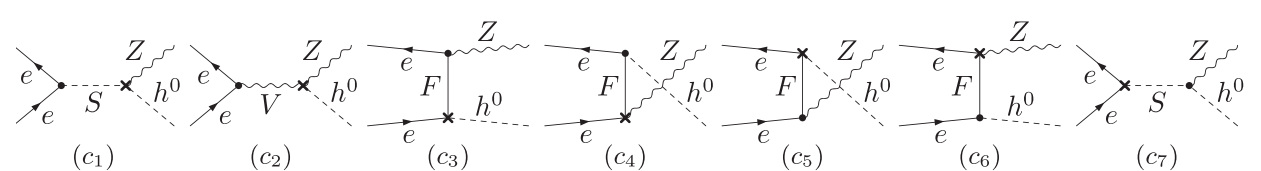

$\left(c_{1}\right)$

$\left(c_{2}\right)$

$\left(c_{3}\right)$

$\left(c_{4}\right)$

$\left(c_{5}\right)$

$\left(c_{6}\right)$

(c)

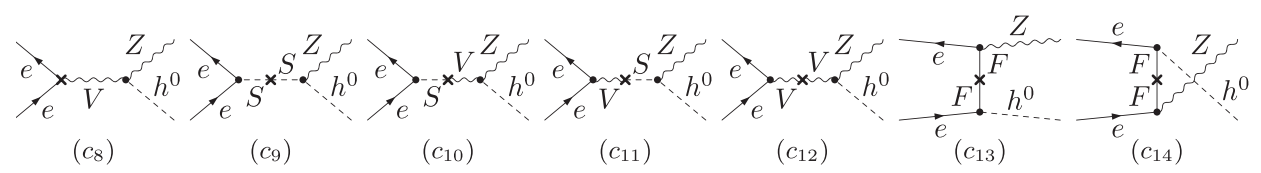

FIG. 6. Generic one-loop counterterms in the 2HDM contributing to the process $e^{+} e^{-} \rightarrow Z h^{0}$ (we take $\phi=h^{0}$ ) are presented. 
TABLE III. NLO SM results in different schemes at $\sqrt{s}=250 \mathrm{GeV}$ are shown (in units of fb). Three schemes are chosen to demonstrate the scale dependence of the results.

\begin{tabular}{lcccrc}
\hline \hline Scheme & $1 / \alpha_{\mathrm{em}}(\mu)$ & $\sigma^{0}$ & $\sigma^{1, \text { weak }}$ & $\sigma^{1}$ & $\sigma^{\mathrm{NLO}}=\sigma^{0}+\sigma^{1}$ \\
\hline$\alpha_{\mathrm{em}}(0)$ & 137.036 & $223.12(0)$ & $6.09(0)$ & $7.13(2)$ & $230.25(2)$ \\
$\alpha_{\mathrm{em}}\left(m_{Z}\right)$ & 128.943 & $252.00(0)$ & $-24.33(0)$ & $-23.07(2)$ & $228.93(2)$ \\
$\alpha_{\mathrm{em}}(\sqrt{s})$ & 127.515 & $257.68(0)$ & $-30.92(0)$ & $-29.63(2)$ & $228.05(2)$ \\
\hline \hline
\end{tabular}

$$
\sigma^{1}=\sigma^{1, \text { weak }}+\sigma^{1, \mathrm{QED}}
$$

Correspondingly, the $\delta$ defined in Eq. (19) can also be divided into two parts:

$$
\delta^{1}=\delta^{1, \text { weak }}+\delta^{1, \mathrm{QED}},
$$

which represent the relative strengths of the weak and QED corrections, respectively.

\section{NUMERICAL RESULTS AND DISCUSSIONS}

Below, we present the numerical results that are obtained from the analysis of the processes $e^{+} e^{-} \rightarrow Z+h / H$ at the one-loop level in both the SM and the $2 \mathrm{HDM}^{3}$ We focus on the following two quantities: the ratio of the weak correction, $\delta^{1 \text {,weak }}$, and the ratio of the full one-loop corrections (including real emissions) to the leading-order results, $\delta^{1}$.

In our numerical calculation, parameters in Table II are used as physical input in the Higgs sector, while in the gauge sector, two different physical parametrizations are used:

1. $\left\{\alpha_{\mathrm{em}}, G_{F}, m_{Z}\right\}$ : These parameters are used in the scan of the 2HDM parameter space, which finally leads to Table II. They are also used in determining the allowed range of $\lambda_{5}$ and in the calculation of the decay part.

2. $\left\{\alpha_{\mathrm{em}}, m_{W}, m_{Z}\right\}$ : These parameters are used in the calculation of production processes, as we have used the on-shell condition for the renormalization of $W$-boson self-energy there.

The values of the above parameters are taken from PDG [95] as $\alpha_{\mathrm{em}}=1 / 137.036, G_{F}=1.16638 \times 10^{-5} \mathrm{GeV}^{-2}$, $m_{Z}=91.1876 \mathrm{GeV}$, and $m_{W}=80.385 \mathrm{GeV}$. It should be noted that the vacuum expectation value (VEV) $v$ is different in these two cases. In the former case, it is determined as $v=1 / \sqrt{\sqrt{2} G_{F}} \approx 246.220 \mathrm{GeV}$, while in the latter case, it is determined as $v=m_{W} \sqrt{1-m_{W}^{2} / m_{Z}^{2}} /$ $\sqrt{\pi \alpha_{\mathrm{em}}}$ and varies as the scale of $\alpha_{\mathrm{em}}$ changes. In the $\alpha_{\mathrm{em}}\left(m_{Z}\right)$ scheme, it is $v \approx 243.137 \mathrm{GeV}$.

\footnotetext{
${ }^{3}$ In the QED part, the extra photon can only attach to an initial electron pair in this process either in the SM or in the 2HDM. Thus, $\delta^{1, \mathrm{QED}}$ should be the same in both the SM and 2HDM results.
}

The total NLO cross section for $e^{+} e^{-} \rightarrow Z h_{\mathrm{SM}}$ including the real emissions can be found in Table III. The results in the $\alpha_{\mathrm{em}}(0)$ scheme still depend on $\log \left(m_{e}\right)$, while the other two are independent. At the LO, the cross sections $\sigma^{0}$ in these three schemes read as 223.12, 252.00, and $257.68 \mathrm{fb}$, respectively, where the maximal difference is $34.56 \mathrm{fb}$. While at NLO, the cross sections $\sigma^{\mathrm{NLO}}$ read as 230.25, 228.93 , and $228.05 \mathrm{fb}$, respectively, where the maximal difference is $1.20 \mathrm{fb}$. From these numbers, we can see that scale dependence has been greatly improved at NLO, which can be exposed more clearly in Fig. 7. For the sake of comparison with other literature, our definition of the weak part agrees with the one defined in Refs. [96,112], and we also find that our numerical results in the SM agree with those given in Refs. [96,112].

In Fig. 7, the dependence on the renormalization scale $\mu$ and the collision energy of the cross section in the SM are explicitly shown. In Fig. 7(a), it is observed that the leading-order results can change drastically when the unphysical renormalization scale $\mu$ varies from 0 to $\sqrt{s}$; i.e., the difference between the results of $\alpha_{\mathrm{em}}(0)$ and $\alpha_{\mathrm{em}}(\sqrt{s})$ can reach up to $15 \%$, as given in Table III. In contrast, the scale dependence of the results is significantly reduced at the next-to-leading order. For example, the difference between the NLO results of $\alpha_{\mathrm{em}}(0)$ and $\alpha_{\mathrm{em}}(\sqrt{s})$ is $1 \%$ or so. In Fig. 7(b), the line shapes of the LO and NLO cross sections varying with the collision energy $\sqrt{s}$ are shown. The largest difference between different renormalization scales occurs near the threshold.

In Fig. 8, we compare the results of leading order and those of the full NLO in the SM. In Fig. 8(a), the distribution of the transverse momentum of the Higgs boson in the process $e^{+} e^{-} \rightarrow Z h$ in the SM is shown where the collision energy $\sqrt{s}$ is taken as $250 \mathrm{GeV}$. When the angle of the outgoing Higgs boson with reference to the incoming electron direction is defined as $\theta$ in the laboratory frame, the transverse momentum of the Higgs boson is defined as $P_{t}(h)=|\vec{P}(h)| \sin \theta$, where $|\vec{P}(h)|$ denotes the magnitude of the three-dimensional momentum of the Higgs boson in the laboratory frame. The cutoff of $P_{t}(h)$ is determined by the total collision energy. When the collision is set to be $250 \mathrm{GeV}$, the maximum of $P_{t}(h)$ is $62.12 \mathrm{GeV}$, which is determined by kinematics. We find that the line shapes of the LO and full NLO results are similar except for a global scaling factor. In Fig. 8(b), the energy dependence of the cross section is shown. 


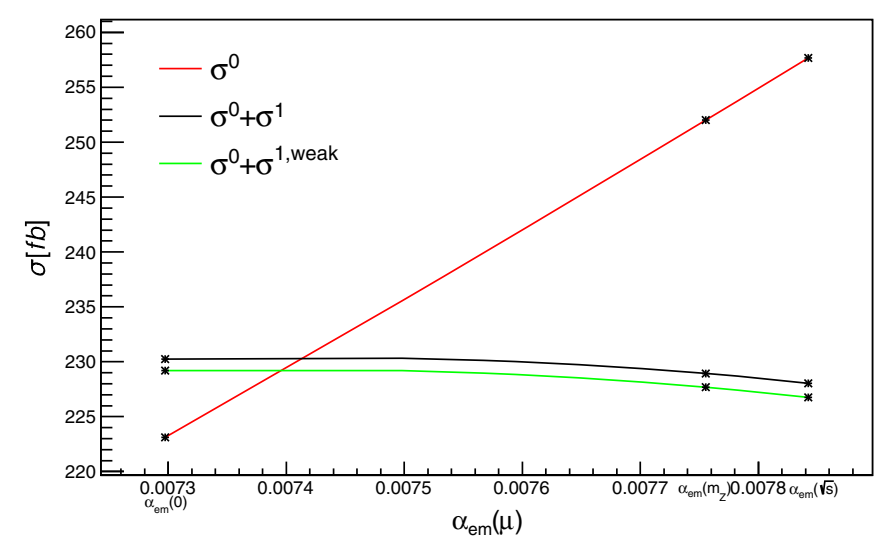

(a)

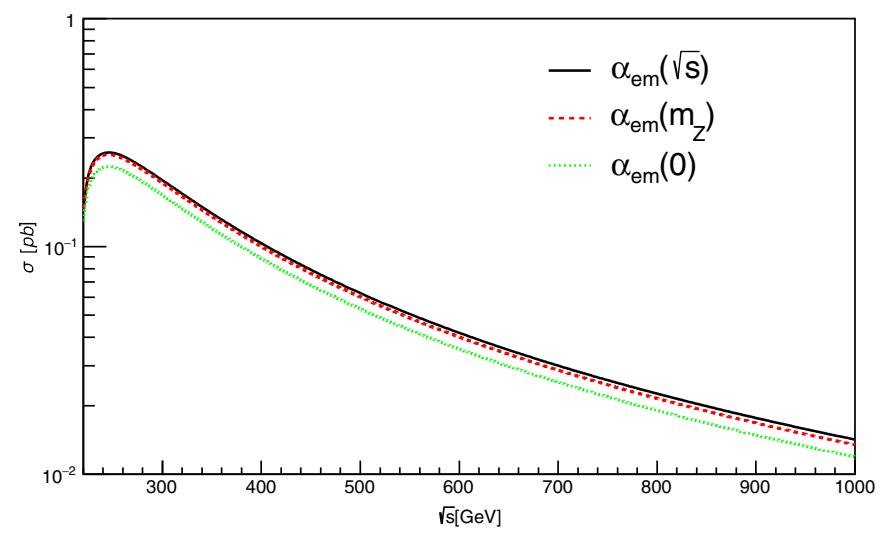

(b)

FIG. 7. (a) Scale dependence of LO and NLO cross section in the SM. (b) LO cross section as a function of collision energy $\sqrt{s}$ in the $\mathrm{SM}$ in different schemes.

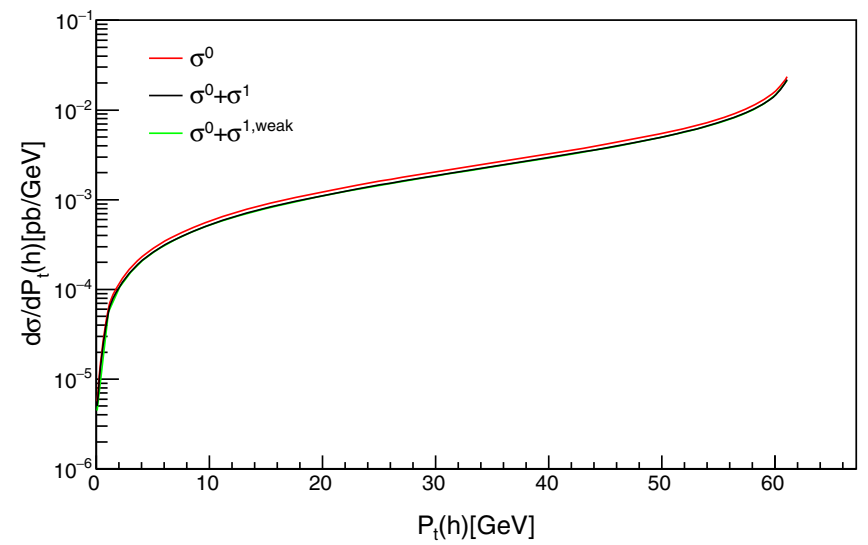

(a)

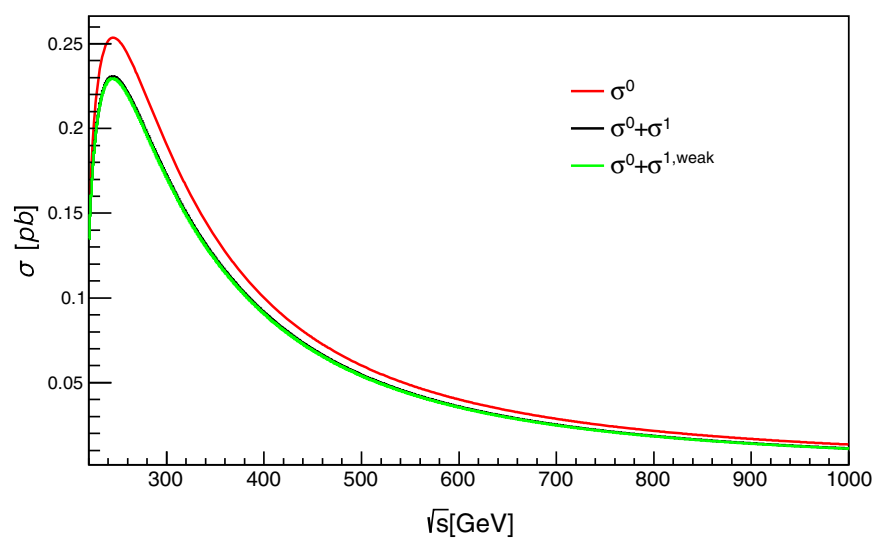

(b)

FIG. 8. (a) Transverse momentum $P_{t}(h)$ distribution of LO and NLO cross section. (b) LO and NLO cross sections as functions of collision energy $\sqrt{s}$ in the SM in the $\alpha_{\mathrm{em}}\left(m_{Z}\right)$ scheme.

As a cross-check, we compare our LO cross section of the SM with that computed using Whizard [113] [say, in the $\alpha_{\mathrm{em}}\left(m_{Z}\right)$ scheme], and a good agreement is found. Moreover, it is observed that although the line shape of $P_{t}(h)$ given in Fig. 8(a) is different from Fig. 2(b) in Ref. [114], where the distribution is a normalized one with the effects of initial-state radiation (ISR) included, a good agreement is found when we also normalize the line shape of $P_{t}(h)$ given in Fig. 8(a) with the tree-level results of Fig. 2(b) of Ref. [114] simulated using MadGraph [115].

Based on the results of the SM, since the NLO results have less dependence on the renormalization scale $\mu$; in the following study on the 2HDM, we will fix a renormalization scale $\mu=m_{Z}$ to present the results.

Now, we start to present the results of the four benchmark points of the $2 \mathrm{HDM}$. The parameter $\lambda_{5}$, which is related to the triple Higgs boson couplings, is given as [48]

$$
\lambda_{5}=\frac{m_{12}^{2}-m_{A}^{2} s_{\beta} c_{\beta}}{v^{2} s_{\beta} c_{\beta}}
$$

Once $\lambda_{5}$ is fixed, all the triple Higgs boson couplings are fixed, as shown in Eqs. (B1)-(B8) of Appendix B. To demonstrate the effects of triple Higgs boson self-couplings, we try to vary it near its value for each benchmark point in Table II. From now on, we treat each BP as a scenario. In each scenario, we fix the mass spectra, $\alpha$, and $\tan \beta$ as given in Table II, and we let $\lambda_{5}$ vary. Due to the constraints from vacuum stability, unitarity, and perturbativity to the theoretical parameters, the parameters $\lambda_{5}$ for the four benchmark scenarios are determined to be in the ranges $[0.545,0.583],[-0.48,0.37],[-1.32,-0.30]$, and $[-6.50,-5.74]$, respectively. We present our results in the $2 \mathrm{HDM}$ with three typical values of $\lambda_{5}$ : the upper bound, the lower bound, and the one in the benchmark point. 
In Fig. 9, the ratios $\delta^{1 \text {,weak }}$ and $\delta^{1}$, which are defined in Eq. (21), are shown, where the results are obtained in the $\alpha_{\mathrm{em}}\left(m_{Z}\right)$ scheme and the label $h$ denotes that the lighter $C P$-even Higgs boson is assumed to be the SM-like Higgs boson. $\delta^{1}$, in which both the weak and QED corrections are included is only presented for the SM; as we have discussed before, $\delta^{1, \mathrm{QED}}$ is exactly the same in both SM and 2HDM. It is found that the signs of the weak part and the QED part are different in the SM. The QED part tends to increase the total cross-section by $+0.5 \sim+1 \%$ as the collision energy increases.

Also, in the BP1-h and BP2-h scenarios, the differences among the curves with different values of $\lambda_{5}$ are small and insignificant. For the BP1-h scenario, this may be attributed to the fact that the allowed range for $\lambda_{5}$ is too narrow and the triple Higgs boson couplings are small, as shown in
Fig. 15(a). In contrast, for the BP2-h scenario, the allowed range is wide enough and the triple Higgs couplings can be large enough, as shown in Fig. 15(b); this small difference instead can be attributed to the decoupling effects of the heavy Higgs boson. It is found that for the BP2-h scenario, this difference from the prediction of the SM is small and typically less than $0.2 \%$ at most, which means that at NLO, new physics plays a less important role. By contrast, for the $\mathrm{BP} 1-\mathrm{h}$ scenario, it is observed that the difference between the new physics and the SM can reach $0.8 \%$ or so from $300 \mathrm{GeV}$ to $500 \mathrm{GeV}$.

In Fig. 10, two benchmark points for an alternative interpretation of the SM-like Higgs boson are considered. In such a scenario, the heavier $C P$-even Higgs boson has a mass near $125 \mathrm{GeV}$. Detailed information on the mass spectra and parameters of these two benchmark points is

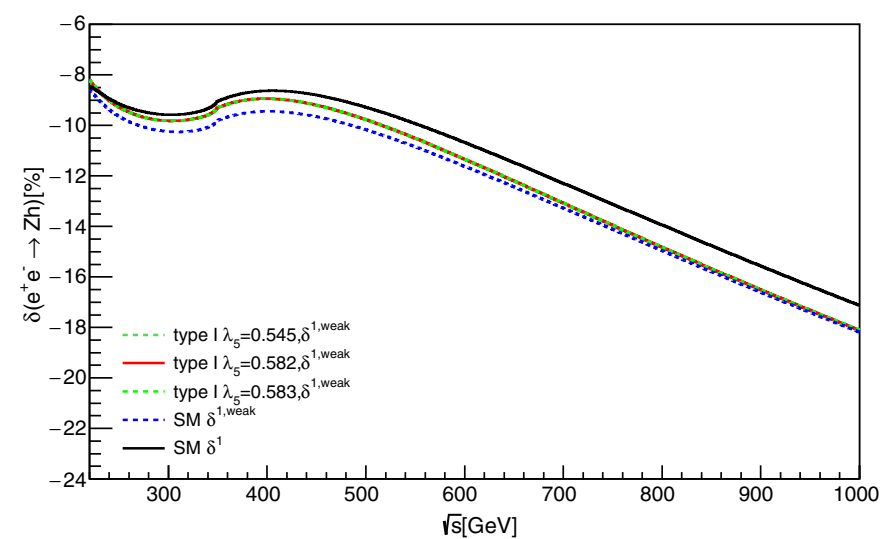

(a) BP1-h

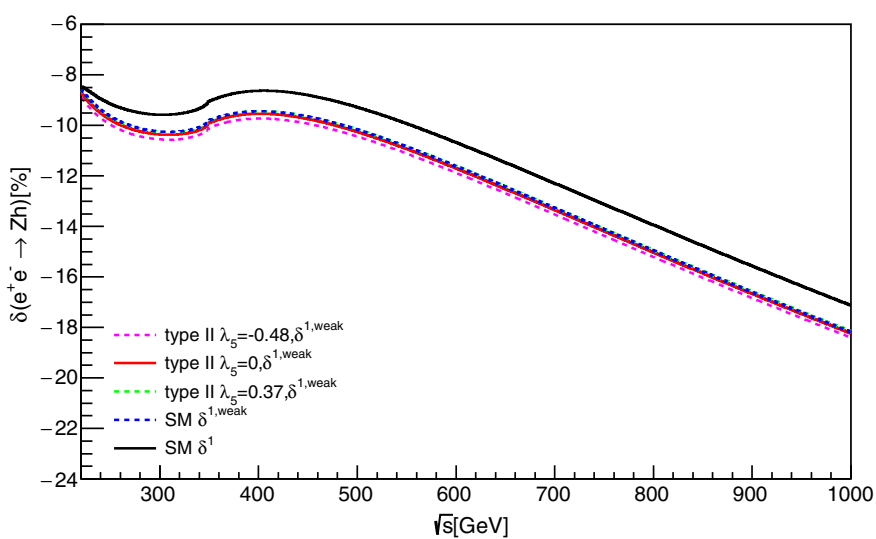

(b) BP2-h

FIG. 9. Ratios of weak and full corrections to LO results for $e^{+} e^{-} \rightarrow Z h$ as functions of collision energy corresponding to benchmark points BP1-h (left) and BP2-h (right) in the 2HDM, where $h^{0}$ is assumed to be the SM-like Higgs boson. A few typical values of $\lambda_{5}$ are taken to show the effects of triple Higgs couplings.

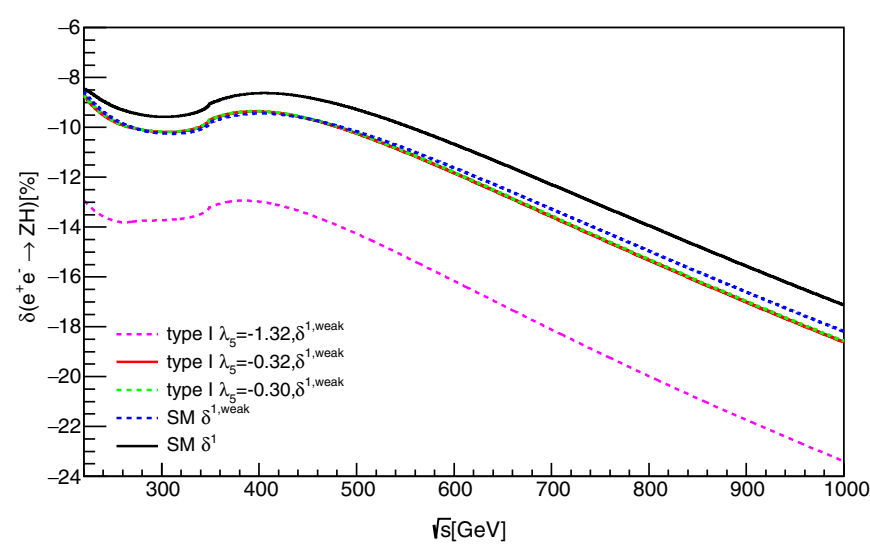

(a) BP1-H

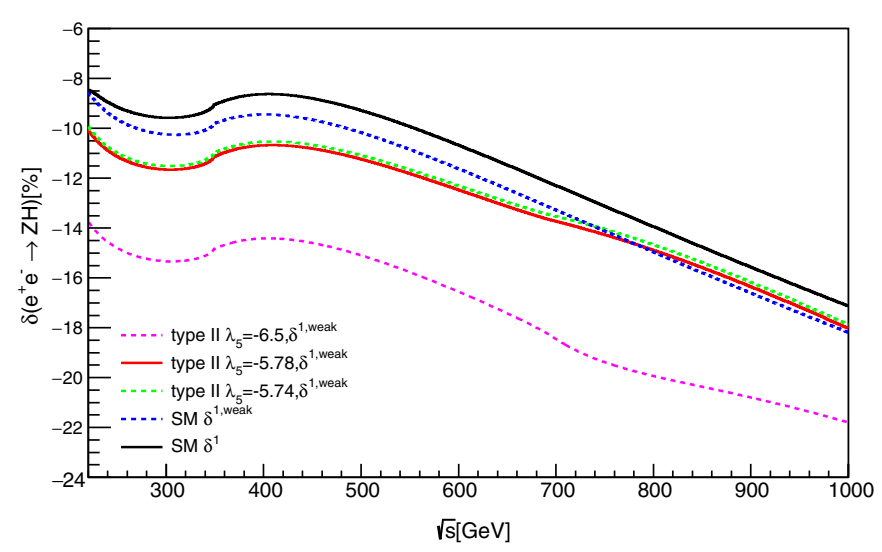

(b) BP2-H

FIG. 10. Ratios of weak and full corrections to LO results for $e^{+} e^{-} \rightarrow Z H$ as functions of collision energy corresponding to benchmark points BP1-H (left) and BP2-H (right) in the 2HDM. A few typical values of $\lambda_{5}$ are taken to show the effects of triple Higgs couplings. 
presented in Table II and labeled as the BP1-H and BP2-H scenarios. Again, three typical values of $\lambda_{5}$ are used to show the effects of new physics. We observe that the ratio for the $\mathrm{BP} 1-\mathrm{H}$ scenario (the pink dashed curve) varies from $-13 \%$ to $-23 \%$ or so, while for the BP2-H scenario it varies from $-13.8 \%$ to $-22 \%$. Also, in this alternative interpretation, the ratio difference for different values of $\lambda_{5}$ (say, $\lambda_{5}=$ -1.32 and $\lambda_{5}=0.30$ for the BP1-H scenario, and $\lambda_{5}=$ -6.5 and $\lambda_{5}=-5.74$ for the BP2-H scenario) can reach more than $5 \%$. It is found that the weak corrections of the 2HDM for these two benchmark points and the typical values of $\lambda_{5}$ have the same sign in the SM, which holds for both the type-I and type-II cases, as well as for the processes $e^{+} e^{-} \rightarrow Z h$ and $e^{+} e^{-} \rightarrow Z H$. Last but not least, all the lines have a bump near the collision energy at 350$400 \mathrm{GeV}$, which can be attributed to the contribution of the top quark pair in the loop functions.

In order to expose the contribution of new physics, in Figs. 11 and 12, we present the contribution of new physics: $\Delta_{Z h}^{\text {weak }}$ and $\Delta_{Z H}^{\text {weak }}$, respectively, with different values of $\lambda_{5}$. The quantity $\Delta^{\text {weak }}$ is defined as

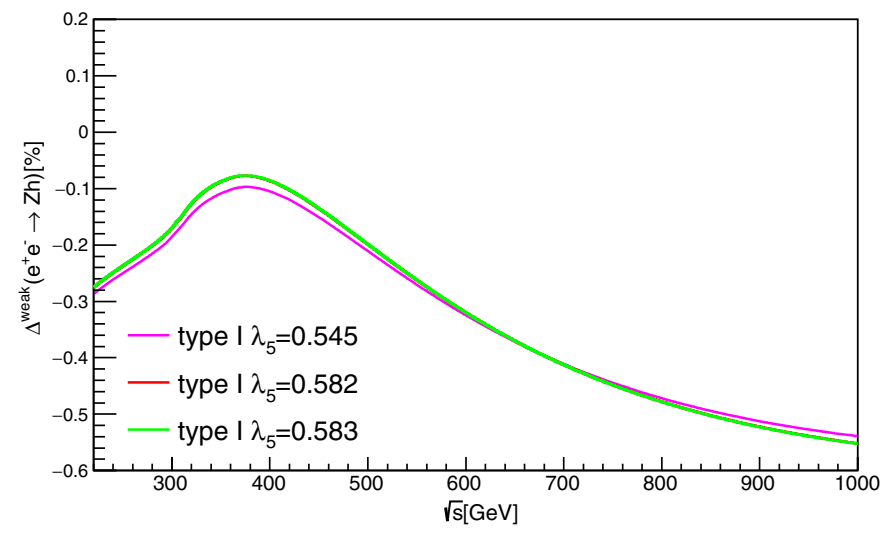

(a) BP1-h

$$
\Delta^{\text {weak }}=\frac{\sigma_{2 \mathrm{HDM}}^{0}+\sigma_{2 \mathrm{HDM}}^{1, \text { weak }}}{\sigma_{\mathrm{SM}}^{0}+\sigma_{\mathrm{SM}}^{1, \mathrm{weak}}}-1,
$$

which describes the contribution of new physics compared with the results of the SM. We have discussed before that $\delta^{1, \mathrm{QED}}$ is exactly the same in both the SM and the 2HDM; thus, $\Delta^{\text {weak }}$ is enough to show the differences. Obviously, in this formula, the pure contribution of the SM is subtracted, while the contributions of new physics and the interference terms between the new physics and the SM are counted.

In Fig. 11, the quantities $\Delta^{\text {weak }}$ of the benchmark scenarios BP1-h and BP2-h are demonstrated. In the left panel, it is observed that the deviations caused by the $2 \mathrm{HDM}$ can change from $-0.28 \%$ to $-0.29 \%$ near the threshold and can decrease to $-0.54 \%$ and $-0.52 \%$ when the collision energy increases to $1000 \mathrm{GeV}$. A bump near $2 m_{H^{ \pm}}=356 \mathrm{GeV}$ can be seen for the BP1-h scenario. In the right panel, the deviations are flat in the explored energy region and can change from $-0.03 \%$ to $-0.37 \%$ at the threshold and can decrease to $+0.02 \%$ and $-0.30 \%$ at

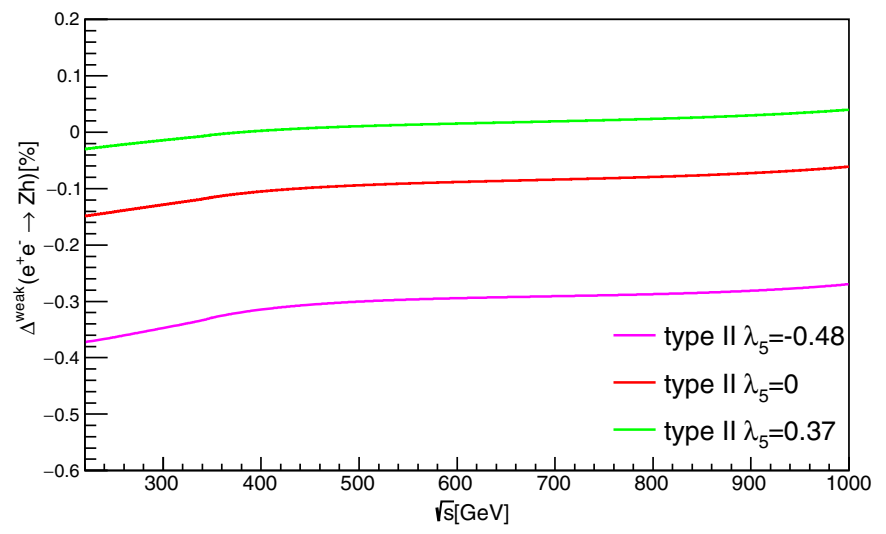

(b) BP2-h

FIG. 11. Ratios of new physics for the process $e^{+} e^{-} \rightarrow Z h$ as functions of collision energy with three typical values of $\lambda_{5}$.

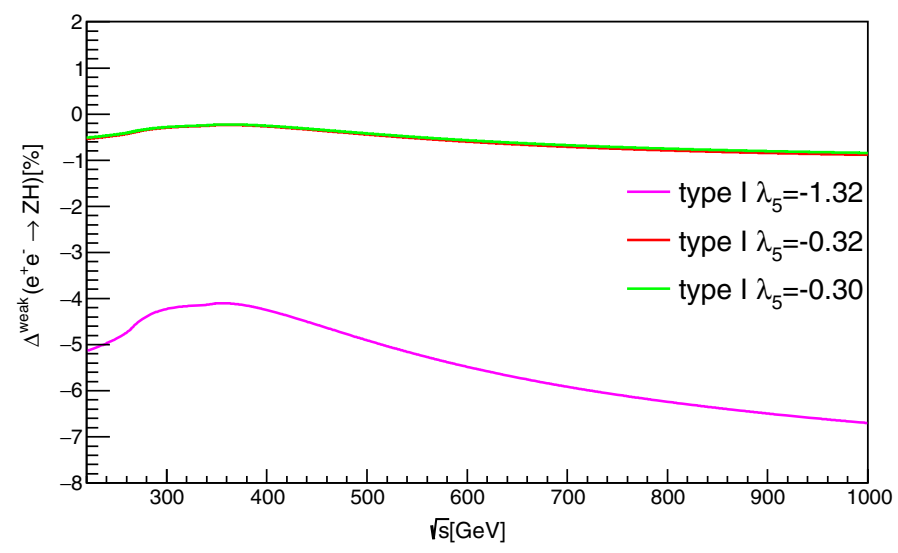

(a) BP1-H

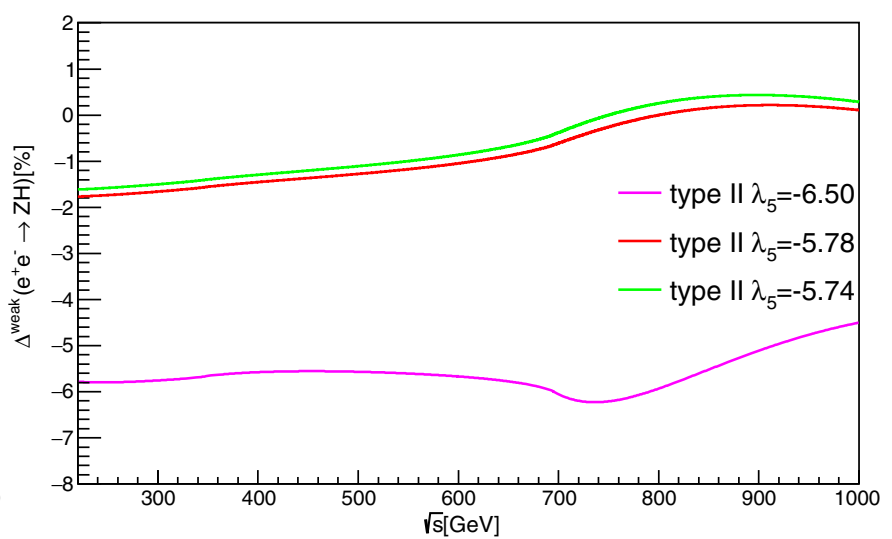

(b) $\mathrm{BP} 2-\mathrm{H}$

FIG. 12. Ratios of new physics for the process $e^{+} e^{-} \rightarrow Z H$ as functions of collision energy with three typical values of $\lambda_{5}$. 
$1 \mathrm{TeV}$. Meanwhile, the deviations are more sensitive to $\lambda_{5}$ in the BP1-h scenario, where new Higgs bosons are relatively lighter than those in the BP2-h scenario, although the allowed range of $\lambda_{5}$ is narrower. For these three cases of the BP2-h scenario, the deviations show no obvious bumps within the explored collision energy.

In Fig. 12, the quantities $\Delta^{\text {weak }}$ of the benchmark scenarios BP1-H and BP2-H are shown. In the left panel, the deviations for the BP1-H scenario are around $-0.58 \%$ at the threshold and are similar for both $\lambda_{5}=-0.32$ and $\lambda_{5}=-0.30$. For the two cases with $\lambda_{5}=-0.32$ and $\lambda_{5}=-0.30$, when the collision energy increases to $1000 \mathrm{GeV}$, the deviations change to $-0.8 \%$ or so. In the case with $\lambda_{5}=-1.32$, the deviation changes from $-5.2 \%$ at the threshold to $-6.6 \%$ at $1000 \mathrm{GeV}$. In the right panel, the deviations for the BP2-H scenario can reach from $-1.6 \%$ to $-5.8 \%$ depending upon the values of $\lambda_{5}$. When the collision energy increases to $1000 \mathrm{GeV}$, the deviations change to $0.1 \%$ to $-4.4 \%$.

For the case of the BP1-H scenario with $\lambda_{5}=-1.32$, a little bump near the energy region $\sqrt{s}=2 m_{H^{ \pm}}=340 \mathrm{GeV}$ can be observed. In contrast, for the two cases of the BP1-H scenario with $\lambda_{5}=-0.30$ and $\lambda_{5}=-0.32$, the new physics contributions show no clear structure with the increase of collision energy due to smaller triple Higgs couplings. For the BP2-H case with $\lambda_{5}=-6.50$, an obvious dip near the energy region $\sqrt{s}=m_{A^{0}}+m_{h^{0}}=695 \mathrm{GeV}$ can be attributed to the contributions of $A^{0}$ and $H^{0}$ via diagrams like $v_{12}, v_{14}, v_{15}$, and $v_{16}$ in Fig. 3 .

Except for the differential cross sections of $e^{+} e^{-} \rightarrow Z \phi_{i}$ for four benchmark points in the 2HDM, it might also be interesting to explore the effects of new physics on the branching fractions of Higgs boson decays, which can be measured to a precision of $1 \%$ or less at future Higgs factories.

The decay width of a neutral Higgs boson in a model including one-loop correction can be formulated as

$$
\begin{aligned}
\Gamma_{1}(\phi \rightarrow f \bar{f})= & \frac{N_{C} G_{F} m_{f}^{2}}{4 \sqrt{2} \pi} \beta_{f}^{3}\left(\xi_{f}^{\phi}\right)^{2} m_{\phi} \hat{Z}_{\phi} \\
& \times\left[1-\Delta r+2 \operatorname{Re}\left(\Delta \mathcal{M}_{1}\right)\right],
\end{aligned}
$$

where $N_{C}$ is the color number, $G_{F}$ is the fermion constant, $m_{f}$ denotes the fermion's mass, $\beta_{f}$ is the speed of the fermion in natural units, and $\xi_{f}^{\phi}$ is the ratio of the couplings of neutral Higgs bosons in the model to those of the SM. $\hat{Z}_{\phi}$ refers to the finite wave function renormalization of the external $\phi$, while $\Delta \mathcal{M}_{1}$ is the amplitude of the one-loop vertex diagrams of $\phi \rightarrow f \bar{f}$. The quantity $\Delta r$ can be found in Ref. [94], which is obviously model dependent and renormalization-scheme dependent. As this part is a work following Ref. [38], the same renormalization scheme as in Ref. [38] is used. Our results for the decays are renormalization-scale dependent, since the $\overline{\mathrm{MS}}$ scheme is used in the renormalization of Higgs boson wave functions.
We have chosen the scale as $\mu_{r}=m_{\phi}$, the mass of the decaying particle.

We can define a quantity $\Delta_{f f}(\phi)$ from Eq. (24) as a deviation from the predictions of the SM, which has the following form:

$$
\begin{aligned}
\Delta_{f f}(\phi) & =\frac{\hat{Z}_{\phi}\left(1-\Delta r^{2 \mathrm{HDM}}+2 \operatorname{Re}\left(\Delta \mathcal{M}_{1}^{2 \mathrm{HDM}}\right)\right)}{\left(1-\Delta r^{\mathrm{SM}}+2 \operatorname{Re}\left(\Delta \mathcal{M}_{1}^{\mathrm{SM}}\right)\right)}-1, \\
f & =b, \tau .
\end{aligned}
$$

More details can be found in Ref. [39].

It is necessary to point out that the value of $\Delta_{f f}(\phi)$ in the $\mathrm{SM}$ is 0 , and it is a measurement of new physics effects at the quantum level. There are several studies which evaluate the BSM effects at the tree-level and one-loop radiative corrections to the decays of Higgs bosons [38], such as the recent Refs. [116,117].

In the type-I and type-II models, the couplings of $h^{0}$ to $b \bar{b}$ and $\tau^{+} \tau^{-}$are proportional to the same factor, $\xi_{f}^{h^{0}}$. It is $\cos \alpha / \sin \beta$ in type I and $-\sin \alpha / \cos \beta$ in type II. The couplings of $H^{0}$ to $b \bar{b}$ and $\tau^{+} \tau^{-}$are also both proportional to the factor $\xi_{f}^{H^{0}}$. In type I, it is $\sin \alpha / \sin \beta$, while in type II, it is $\cos \alpha / \cos \beta$. Obviously, there exist two special cases: 1) the couplings $\left|\xi_{f}^{h^{0}}\right|=1$ if $h^{0}$ corresponds to the SMlike Higgs boson, and 2) the couplings $\left|\xi_{f}^{H^{0}}\right|=1$ if $H^{0}$ corresponds to the SM-like Higgs boson. Apparently, from tree-level expression, it is impossible to separate these cases from the SM. Below, we explore whether it is possible to distinguish these cases by using the quantum corrections-i.e., by resorting to the information of $\Delta_{f f}\left(h^{0}\right)$ and $\Delta_{f f}\left(H^{0}\right)$.

We use HiggsSignals [65-67], which incorporates the simplified template cross section (STXS) framework [118] to evaluate the $\chi^{2}$ of each point in the parameter space. The $\chi^{2}$ of HiggsSignals we used here includes two parts: 1) a signal strength, $\chi_{\mu}^{2}$, and 2) a mass peak, $\sum_{i} \chi_{m_{i}}^{2}$. In the signal strength part, when $N$ observables for a given Higgs boson are considered, the $\chi_{\mu}^{2}$ (here $\mu$ denotes one of Higgs bosons) equals $\chi_{\mu}^{2}=\sum_{\alpha=1}^{N} \chi_{\mu, \alpha}^{2}=(\hat{\mu}-\mu)^{T} C_{\mu}^{-1}(\hat{\mu}-\mu)$, where $(\hat{\mu}-\mu)$ should be understood as an $N$-dimensional vector and $C_{\mu}$ is the $N \times N$ signal strength covariance matrix. Theoretical observables in the $\chi^{2}$ of HiggsSignals are defined as signal strengths, $\hat{\mu}$. When the final states of Higgs decay are specified, a signal strength is defined as $\hat{\mu}=\sum_{i}\left(\sigma_{i}^{2 \mathrm{HDM}} B r^{2 \mathrm{HDM}}\right) /\left(\sigma_{i}^{\mathrm{SM}} B r^{\mathrm{SM}}\right)$, where $\sigma_{i}$ denotes the cross section of a production process and $\mathrm{Br}$ denotes the decay branching fraction of the Higgs boson. The mass peak part takes into account all neutral Higgs bosons in a model, which is defined as $\chi_{m_{i}}^{2}=\sum_{\alpha=1}^{N} \chi_{m_{i}, \alpha}^{2}=\left(\hat{m}-m_{i}\right)^{T} \times$ $C_{m_{i}}^{-1}\left(\hat{m}-m_{i}\right)$, where $N$ neutral Higgs bosons are assumed. In our study of $2 \mathrm{HDM}$, the $\chi^{2}$ mainly takes into account $\chi_{\mu}^{2}$ and the $\chi_{m_{i}}^{2}$, where $m_{i}$ is assigned to be the SM-like Higgs 
boson. For example, when $H^{0}$ is assigned to be the SM-like Higgs boson in the BP1-H and BP2-H scenarios, the corresponding $\chi_{\mu}^{2}$ and $\chi_{m_{H^{0}}}^{2}$ are computed.

In Figs. 13 and 14, in order to examine $\Delta_{f f}(\phi)$ in the 2HDM, we use the mass parameters from Table II and $\left|\xi_{f}^{\phi}\right|=1$ to fix $\alpha$. Then we allow two parameters- $\tan \beta$ and $\lambda_{5}$ - to change. These two parameters are allowed to vary within the ranges $2<\tan \beta \leq 15$ and $-6 \leq \lambda_{5} \leq 1$ while only points which satisfy all theoretical and experimental constraints are accepted. In Fig. 13, we examine numerically $\Delta_{b b}(h)$ and $\Delta_{\tau \tau}(h)$ for the D1-h and D2-h scenarios, where $h^{0}$ is assumed to be the SM-like Higgs boson. We use D1-h and D2-h to label these two different scenarios which have the same mass spectra as the BP1-h and BP2-h scenarios in Table II, respectively. In this practice, it is obvious that the sign of $\cos (\beta-\alpha)$ is allowed to vary; therefore, we assume it to be either positive or negative. In Fig. 14, we examine $\Delta_{b b}(H)$ and $\Delta_{\tau \tau}(H)$ for the D1-H and D2-H scenarios, where $H^{0}$ is assumed to be the SM-like Higgs boson; likewise, the D1-H and D2-H scenarios share the same mass spectra as $\mathrm{BP} 1-\mathrm{H}$ and $\mathrm{BP} 2-\mathrm{H}$, respectively. Meanwhile, the sign of $\sin (\beta-\alpha)$ is allowed to be both
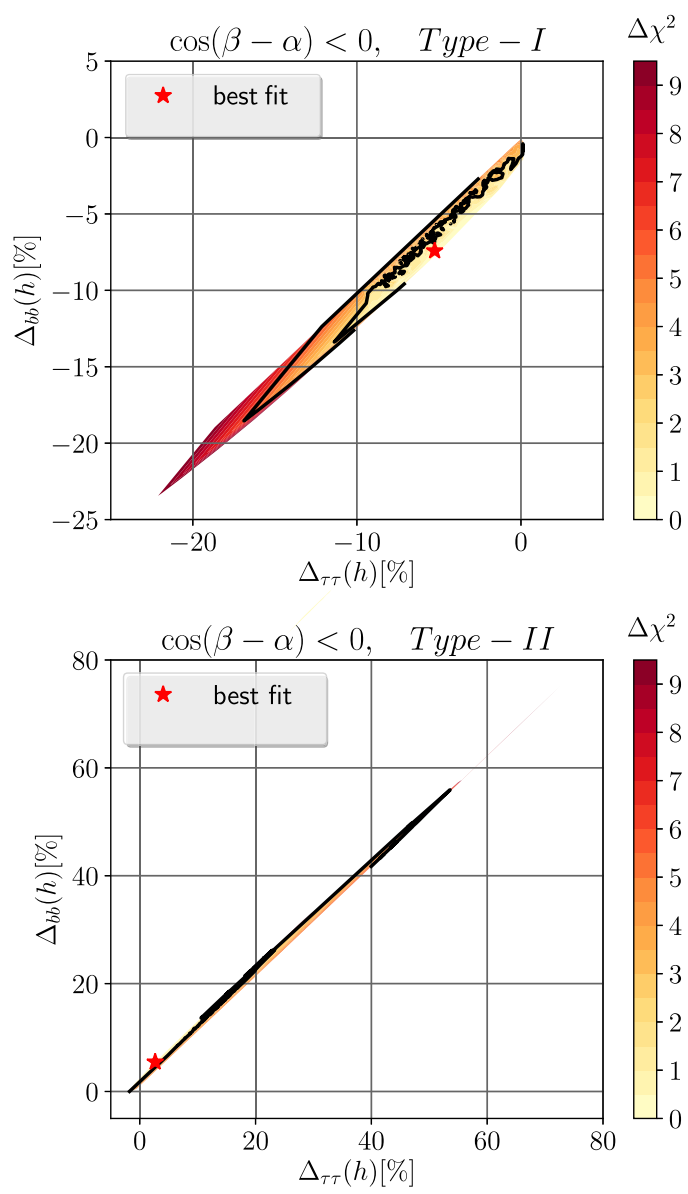

positive and negative, so we label the scenarios as $\pm \mathrm{D} 1-\mathrm{H}$ and $\pm \mathrm{D} 2-\mathrm{H}$. Similarly, we label other scenarios as $\pm \mathrm{D} 1$ - $\mathrm{h}$ and \pm D2-h. In total, we can have eight scenarios.

By scanning over the parameter space of $2 \mathrm{HDM}$, we can compute its corresponding $\chi^{2}$ value. Obviously, the $\chi^{2}$ can be determined for each point in the parameter space, and then the difference of $\Delta \chi^{2}=\chi^{2}-\chi_{\text {min }}^{2}$ can be computed. The total of number of freedoms $\nu$ is defined as $\nu=n_{o}-n_{p}$, where $n_{o}$ is the number of observables taken from experiment measurements (which is equal to 78 in this work) and $n_{p}$ is the number of model parameters in the 2HDM (which is equal to 7). For a general fit, as shown in Fig. 1 and the results provided in Table II, the number of freedoms is $78-7=71$. Now, for the specific fits shown in Figs. 13 and 14, due to the constraint of $\left|\xi_{f}^{\phi}\right|=1$ and the fact that we have fixed four mass parameters, there are only two free parameters. Therefore, the degree of freedom is $78-2=76$.

In Figs. 13 and 14, we show points that have satisfied the condition $\Delta \chi^{2} \leq 11$ ( $<3 \sigma$ for a two-parameter fit). For all eight scenarios (labeled as \pm Dh-1, \pm Dh-,$\pm \mathrm{DH}-1$, and $\pm \mathrm{DH}-2)$, the main $\Delta \chi^{2}$ is mainly the contributions of $\chi_{\mu}^{2}$ from the signal-strength observables, since $\chi^{2}$ of the mass
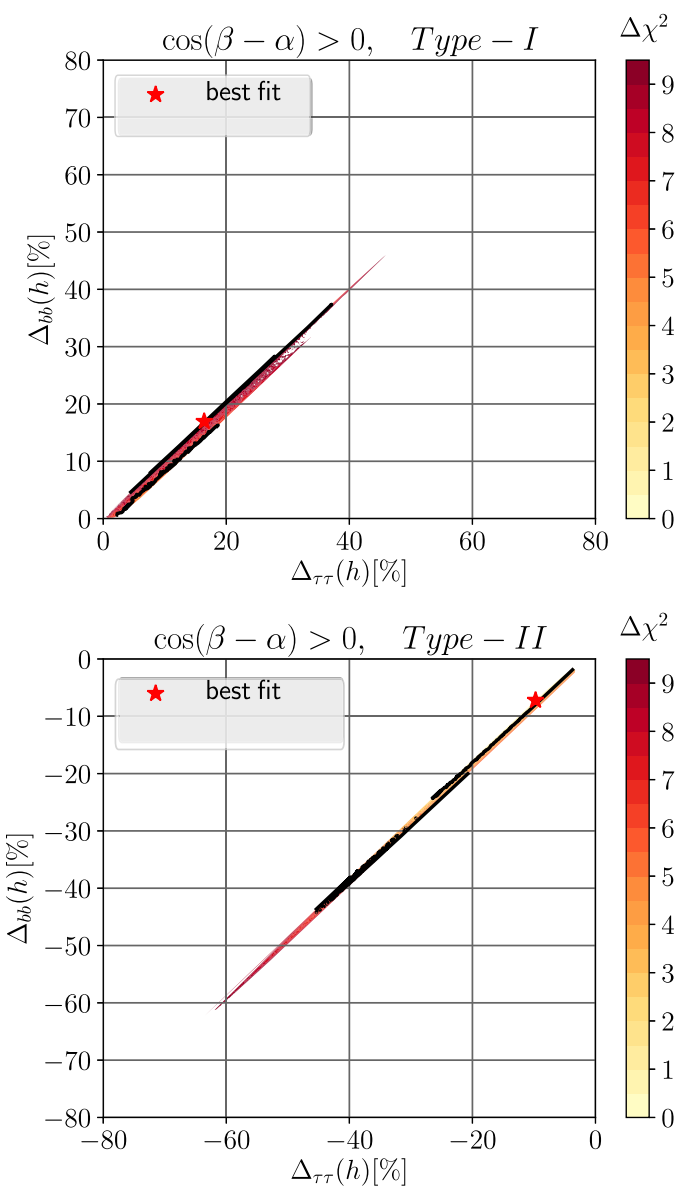

FIG. 13. Correlations between relative precisions $\Delta_{\tau \tau}(h)$ and $\Delta_{b b}(h)$ for the 2 HDM, where $h^{0}$ is assumed to be the SM-like Higgs boson with $m_{H}=212 \mathrm{GeV}$ for the $\pm \mathrm{D} 1$-h scenarios (upper panels) and $m_{H}=594 \mathrm{GeV}$ for the $\pm \mathrm{D} 2$-h scenarios (lower panels). 

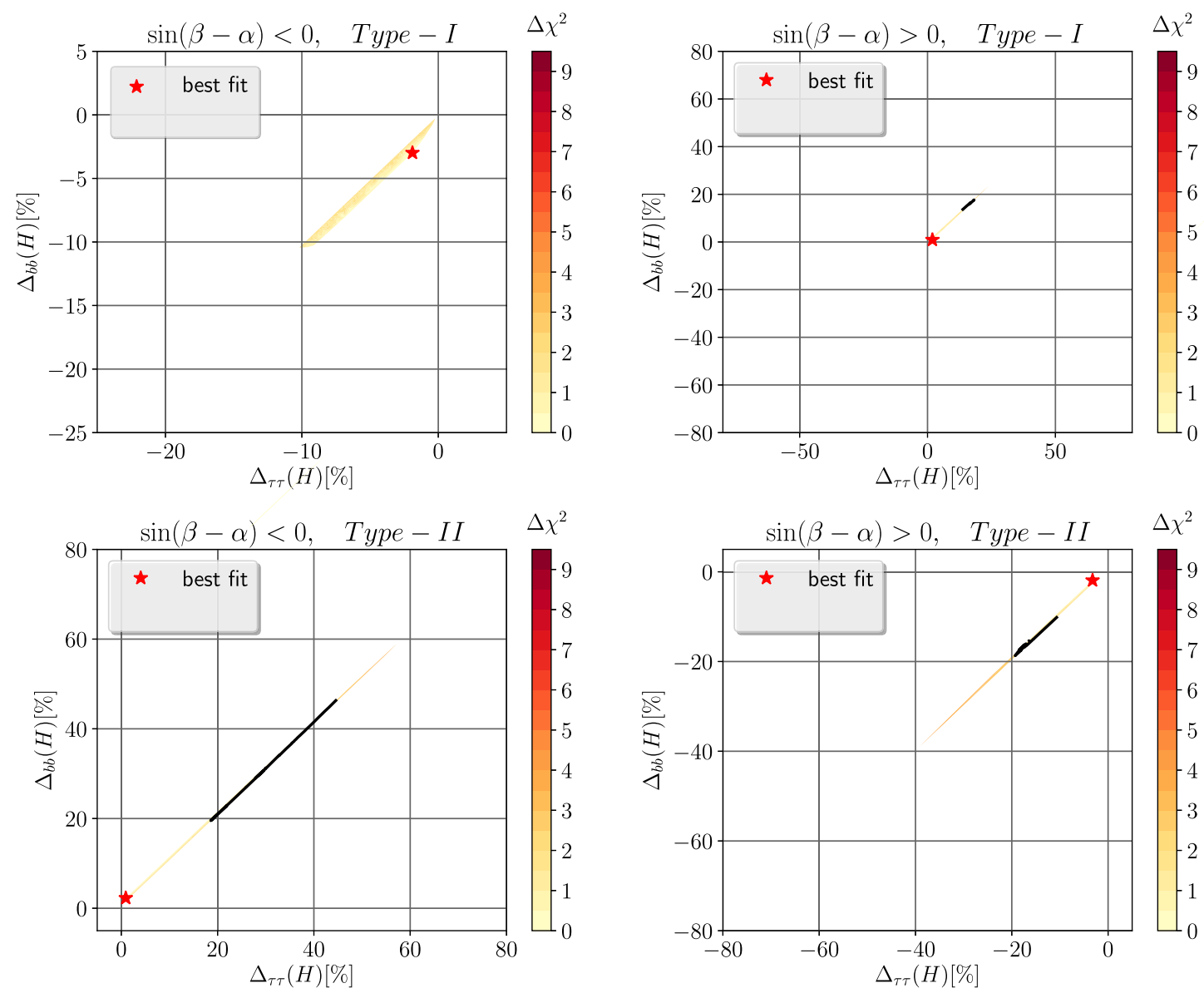

FIG. 14. Correlation between relative precisions $\Delta_{\tau \tau}(H)$ and $\Delta_{b b}(H)$ for the 2HDM, where $H^{0}$ is assumed to be the SM-like Higgs boson. The coding color is the same as in Fig. 13, with $m_{h}=95 \mathrm{GeV}$ for the $\pm \mathrm{D} 1-\mathrm{H}$ scenarios (upper panels) and the $\pm \mathrm{D} 2-\mathrm{H}$ scenarios (lower panels).

part is tiny and negligible. Plots are shown up to $99 \%$ C.L. It is remarkable that our results are in good agreement with previous publications $[39,119]$. There are a few comments:

1. A positive correlation between $\Delta_{\tau \tau}(h)$ and $\Delta_{b b}(h)$ is observed for both $\pm \mathrm{D} 1$-h and $\pm \mathrm{D} 2$-h, as demonstrated in Fig. 13, where the color bar indicates regions close to the minimum $\chi^{2}$. The best-fit point is indicated by a red star in each of these figures.

It turns out that in the D1-h scenarios, the corrections to $\Delta_{b b}(h)$ and $\Delta_{\tau \tau}(h)$ depend on the sign of $\cos (\beta-\alpha)$; i.e., when $\cos (\beta-\alpha)>0$ $[\cos (\beta-\alpha)<0]$, the corrections are positive (negative). It can deviate from the prediction of the SM by $+15 \%(-6 \%)$ for the best-fit point. In contrast, the opposite is the case for the BP2-h scenario, and it is allowed to deviate from the prediction of the SM by less than $-6 \%(+5 \%)$.

2. An interesting observation is that due to the positive correlation between $\Delta_{b b}(h)$ and $\Delta_{\tau \tau}(h)$, it is possible to determine the sign of $\cos (\beta-\alpha)$ by using precise measurements of the branching fractions of $h \rightarrow b \bar{b}$ and $h \rightarrow \tau \tau$. As pointed out in Refs. [39,120-122], if the couplings of Higgs to fermions can be determined up to $8 \%$, it becomes possible to discriminate the types of Yukawa interactions.

3. At the CEPC or the ILC $[15,22,123]$, the precision of Higgs coupling measurements can typically reach $\mathcal{O}(1) \%$, and there is no doubt on their capability to discriminate the types of Yukawa interactions, like the four scenarios introduced here.

The deviations of $H$ decaying into $b \bar{b}$ and $\tau \tau$ are presented in Fig. 14, where the correlations of $\Delta_{b b}(H)$ and $\Delta_{\tau \tau}(H)$ for the $\pm \mathrm{D} 1-\mathrm{H}$ and $\pm \mathrm{D} 2-\mathrm{H}$ scenarios are demonstrated. One remarkable difference from Fig. 13 is that the allowed deviations of $\Delta_{b b}(H)$ and $\Delta_{\tau \tau}(H)$ from those of the SM are smaller than $2.5 \%$ and $-3 \%$, respectively, for the best-fit point. Such a deviation is even smaller (less than 1\%), as shown by BP2-H, in the D2-H scenarios. Such deviations are quite distinctive due to Yukawa structure in these four scenarios. In other words, when a deviation is observed, an upper limit can be set on the mixing angles $\cos (\beta-\alpha)$ or $\sin (\beta-\alpha)$. 


\section{DISCUSSIONS AND CONCLUSION}

In this work, we have proposed four benchmark scenarios of the 2HDM after taking into account the current Higgs data from the LHC. We have evaluated the radiative corrections to the process $e^{+} e^{-} \rightarrow Z \phi$ in the SM and these four benchmark scenarios up to one-loop level.

In the Monte Carlo simulation results [124] obtained using Whizard, it is found that the ISR can decrease the total LO cross section of $e^{+} e^{-} \rightarrow Z h$ in the SM by more than $10 \%$ when the contributions of high-order logarithms are resummed. In contrast, the real emission in this work can increase the total LO cross section by only a factor of $+0.5 \%$. This simply manifests the difference between the resummed and the fixed-order results. Meanwhile, we find that the one-loop weak correction of the SM can reduce the total NLO cross section by $9.15 \%$, as demonstrated in Table III.

As an estimation, we can express the total cross section with ISR as

$$
\sigma^{\mathrm{LO}}(s)=\bar{\sigma}^{W, \mathrm{LO}}(s) R\left(1-\frac{\Delta E}{E}, s\right),
$$

which had been proposed by Ref. [125] when $\frac{\Delta E}{E} \ll 1$ ( $E$ denotes the energy of the electron/positron, and $\Delta E$ is the energy of the soft photon), where $R\left(1-\frac{\Delta E}{E}, s\right)$ denotes the convolution of incoming fluxes of electrons and positrons (i.e., ISR), and $\bar{\sigma}^{\mathrm{LO}}(s)$ denotes the cross section without weak corrections. It should be pointed out that $\bar{\sigma}^{W, \mathrm{LO}}$ has included high-order QED corrections but no weak corrections. The numerical results given in Ref. [124] demonstrate that $R\left(1-\frac{\Delta E}{E}, s\right)$ is roughly equal to 0.90 .

When the one-loop weak correction is taken into account, the total cross section at NLO with ISR can be expressed as

$$
\sigma^{\mathrm{NLO}}(s)=\bar{\sigma}^{W, \mathrm{NLO}}(s) R\left(1-\frac{\Delta E}{E}, s\right),
$$

where $R\left(1-\frac{\Delta E}{E}, s\right)$ (i.e., ISR) is the same as in the LO case. Our results demonstrate that weak correction can reduce $\bar{\sigma}^{W, \mathrm{LO}}$ by a factor of $9.15 \%$ with $\mu=m_{Z}$. We can use the NLO cross section $\bar{\sigma}^{W, \mathrm{NLO}}=228 \mathrm{fb}$ with $\mu=m_{Z}$ to estimate the total cross section with both ISR and weak corrections, which yields $\sigma^{\mathrm{NLO}}(s)=228 \times 0.9=205.2 \mathrm{fb}$, which is $18.6 \%$ smaller than the tree-level cross section in the $\alpha_{\mathrm{em}}\left(m_{Z}\right)$ scheme given in Table III.

Here we add one more comment on the ISR. It is well known that due to the soft collinear divergences of QED, the large logarithmic terms in the form of $\alpha_{\mathrm{em}}^{n} \log ^{n} \frac{s}{m_{e}^{2}} \log ^{m} \frac{m_{e}}{\lambda}$ (where $\lambda$ denotes the mass of the photon or IR cutoff) from the ISR should be resummed. The effects of ISR at electron-positron colliders can affect cross sections of physics processes significantly [126,127]. In
Whizard [113], the effect of ISR to all orders [125,128,129] has been implemented in the structure functions of incoming electrons and positrons. The ISR has been implemented in other Monte Carlo codes [127,130-133] and applied to LEP I [134] and LEP II [135] experimental analysis. Therefore, it is expected that the results for the SM from Whizard with ISR are more accurate in capturing large logs. However, for the study of the 2HDM, currently the ISR effect has not been implemented in whizard, and therefore there is no alternative code with which to check it.

We compute the decays $\phi \rightarrow b \bar{b}$ and $\phi \rightarrow \tau^{+} \tau^{-}$with $\phi=$ $h^{0}\left(H^{0}\right)$ in Dh-1 and Dh-2 (DH-1 and DH-2) by including the EW corrections. We have shown that in the Dh-2 and DH-2 scenarios, the electroweak radiative corrections in these two decay processes are rather small due to the fact that the heavy states $A^{0}, H^{0}$, and $H^{ \pm}$have masses of the order of $600 \mathrm{GeV}$, while they could be sizeable for D1-h and D1-H, as shown in Figs. 13 and 14. Considering the recent progress in the gauge-independent renormalization schemes $[101,104,107]$, it will be interesting to evaluate the differences caused by different renormalization schemes.

Our results demonstrate that $e^{+} e^{-}$colliders (especially the Higgs factories with $\sqrt{s}=250 \mathrm{GeV}$ ) can offer us the potential to distinguish various $2 \mathrm{HDM}$ models by looking at quantum effects in Higgs observables. Except for performing precision measurements of the SM-like Higgs boson, linear colliders also have the potential to discover new physics. For example, it can directly produce the light charged Higgs boson pair in BP1-h and BP1-H scenarios via $e^{+} e^{-} \rightarrow H^{+} H^{-}$processes [136,137]. The energy scan of $e^{+} e^{-}$colliders can also help to detect the mass spectra of Higgs bosons and even triple couplings of Higgs bosons, as shown in Figs. 11 and 12. If nature chooses $2 \mathrm{HDM}$ as the new physics beyond the SM in the $\mathrm{TeV}$ region, it would be hopeful to probe parameters of the Higgs potential sector by using the data of productions and decays. The results of the proposed benchmark points show that precision measurement on the cross sections at different collision energies is helpful for exploring the mass spectra and Higgs couplings of the 2HDM.

\section{ACKNOWLEDGMENTS}

We thank Abdesslam Arhrib and David Lopez-Val for helpful discussion at the early stage of this work. This work is supported by the Moroccan Ministry of Higher Education and Scientific Research MESRSFC and CNRST: Project No. PPR/2015/6. R. B. was supported in part by the Chinese Academy of Sciences (CAS) President, International Fellowship Initiative (PIFI) program (Grant No. 2017VMB0021). Q. S. Yan is supported by the Natural Science Foundation of China under Grants No. 11475180 and No. 11875260. B. Gong is supported by the Natural Science Foundation of China under Grants No. 11475183 and No. 11975242. 


\section{APPENDIX A: THE IR BEHAVIOR OF $e^{+} e^{-} \rightarrow Z h$ IN THE SM}

The dependence of the corrections at NLO, $\sigma^{1}$, on $\Delta E$ and $\Delta \theta$ are shown in Tables IV and V. In Table IV, the dependence is seen in a wide range, and we choose $\delta_{s}=10^{-3}$ as our default choice. In Table $\mathrm{V}$, the result becomes cut-dependent when $\Delta \theta$ is smaller than $10^{-4}$. It is because the approximation in Eq. (14) demands $\Delta \theta \gg m_{e} / \sqrt{s} \sim 2 \times 10^{-6}$. Thus, we choose $\Delta \theta=10^{-3}$ in our setting.

One-loop radiation corrections include collinear singularities which can be infinite when $m_{e}$ goes to zero, and they become terms proportional to $\log \left(m_{e}\right)$ in this limit. Some of these terms are canceled when virtual and real corrections are summed up, and some of them can be absorbed into the redefinition of running coupling constant as mentioned above, but the collinear singularities cannot be removed completely, which demands a careful manipulation.

To deal with these remaining collinear singularities, we used the following fixed-order electron structure function, which can be derived from Eq. (11) of Ref. [125]:

$$
f_{e e}(x, s)=\delta(1-x)+\frac{\alpha_{\mathrm{em}}}{2 \pi} \log \frac{s}{4 m_{e}^{2}} P_{e e}^{+}(x, 0),
$$

TABLE IV. Check for $\Delta E$-independence at $\sqrt{s}=250 \mathrm{GeV}$ (in units of $10^{-1} \mathrm{pb}$ ).

\begin{tabular}{lccccc}
\hline \hline$\delta_{s}=2 \Delta E / \sqrt{s}$ & $\sigma_{S+V}$ & $\sigma_{H \bar{C}}$ & $\sigma_{\mathrm{HC}+\mathrm{CT}}^{*}$ & $\sigma_{\mathrm{SC}}$ & $\sigma^{1}$ \\
\hline $10^{-1}$ & $-0.7127(0)$ & $0.1240(0)$ & $-0.1209(0)$ & $0.4794(0)$ & $-0.2302(0)$ \\
$10^{-2}$ & $-1.4347(0)$ & $0.5445(0)$ & $-0.5306(1)$ & $1.1903(0)$ & $-0.2305(1)$ \\
$10^{-3}$ & $-2.1567(0)$ & $0.9788(1)$ & $-0.9540(2)$ & $1.9012(0)$ & $-0.2307(2)$ \\
$10^{-4}$ & $-2.8787(0)$ & $1.4142(1)$ & $-1.3784(2)$ & $2.6121(0)$ & $-0.2308(2)$ \\
$10^{-5}$ & $-3.6006(0)$ & $1.8497(2)$ & $-1.8027(4)$ & $3.3230(0)$ & $-0.2306(4)$ \\
$10^{-6}$ & $-4.3227(0)$ & $2.2853(2)$ & $-2.2271(5)$ & $4.0339(0)$ & $-0.2306(5)$ \\
$10^{-7}$ & $-5.0446(0)$ & $2.7208(2)$ & $-2.6516(6)$ & $4.7448(0)$ & $-0.2306(6)$ \\
$10^{-8}$ & $-5.7666(0)$ & $3.1564(3)$ & $-3.0762(7)$ & $5.4558(0)$ & $-0.2306(8)$ \\
\hline \hline
\end{tabular}

TABLE V. Check for $\Delta \theta$-independence at $\sqrt{s}=250 \mathrm{GeV}$ (in units of $10^{-1} \mathrm{pb}$ ).

\begin{tabular}{lccccc}
\hline \hline$\Delta \theta$ & $\sigma_{V+S}$ & $\sigma_{H \bar{C}}$ & $\sigma_{\mathrm{HC}+\mathrm{CT}}^{*}$ & $\sigma_{\mathrm{SC}}$ & $\sigma^{1}$ \\
\hline $10^{-1}$ & $-2.1567(0)$ & $0.3856(1)$ & $-0.3608(1)$ & $1.9012(0)$ & $-0.2307(1)$ \\
$10^{-2}$ & $-2.1567(0)$ & $0.6822(1)$ & $-0.6574(1)$ & $1.9012(0)$ & $-0.2307(1)$ \\
$10^{-3}$ & $-2.1567(0)$ & $0.9788(1)$ & $-0.9540(2)$ & $1.9012(0)$ & $-0.2307(2)$ \\
$10^{-4}$ & $-2.1567(0)$ & $1.2751(1)$ & $-1.2506(3)$ & $1.9012(0)$ & $-0.2310(3)$ \\
$10^{-5}$ & $-2.1567(0)$ & $1.5527(1)$ & $-1.5471(3)$ & $1.9012(0)$ & $-0.2499(3)$ \\
$10^{-6}$ & $-2.1567(0)$ & $1.6227(2)$ & $-1.8437(4)$ & $1.9012(0)$ & $-0.4765(4)$ \\
\hline \hline
\end{tabular}

TABLE VI. Check for $m_{e}$-independence at $\sqrt{s}=250 \mathrm{GeV}$ (in units of $10^{-1} \mathrm{pb}$ ).

\begin{tabular}{lccccc}
\hline \hline$k$ & $\sigma_{V+S}$ & $\sigma_{H \bar{C}}$ & $\sigma_{\mathrm{HC}+\mathrm{CT}}^{*}$ & $\sigma_{\mathrm{SC}}$ & $\sigma^{1}$ \\
\hline $2^{-4}$ & $-2.5815(0)$ & $0.9788(1)$ & $-0.9540(2)$ & $2.3260(0)$ & $-0.2307(2)$ \\
$2^{-3}$ & $-2.4753(0)$ & $0.9788(1)$ & $-0.9540(2)$ & $2.2198(0)$ & $-0.2307(2)$ \\
$2^{-2}$ & $-2.3691(0)$ & $0.9788(1)$ & $-0.9540(2)$ & $2.1136(0)$ & $-0.2307(2)$ \\
$2^{-1}$ & $-2.2629(0)$ & $0.9788(1)$ & $-0.9540(2)$ & $2.0074(0)$ & $-0.2307(2)$ \\
$2^{0}$ & $-2.1567(0)$ & $0.9788(1)$ & $-0.9540(2)$ & $1.9012(0)$ & $-0.2307(2)$ \\
$2^{1}$ & $-2.0505(0)$ & $0.9788(1)$ & $-0.9540(2)$ & $1.7950(0)$ & $-0.2307(2)$ \\
$2^{2}$ & $-1.9443(0)$ & $0.9788(1)$ & $-0.9540(2)$ & $1.6888(0)$ & $-0.2307(2)$ \\
$2^{3}$ & $-1.8381(0)$ & $0.9788(1)$ & $-0.9540(2)$ & $1.5826(0)$ & $-0.2307(2)$ \\
$2^{4}$ & $-1.7318(0)$ & $0.9788(1)$ & $-0.9540(2)$ & $1.4763(0)$ & $-0.2307(2)$ \\
$2^{5}$ & $-1.6256(0)$ & $0.9788(1)$ & $-0.9540(2)$ & $1.3701(0)$ & $-0.2307(2)$ \\
$2^{6}$ & $-1.5194(0)$ & $0.9788(1)$ & $-0.9540(2)$ & $1.2639(0)$ & $-0.2307(2)$ \\
$2^{7}$ & $-1.4132(0)$ & $0.9788(1)$ & $-0.9540(2)$ & $1.1577(0)$ & $-0.2307(2)$ \\
$2^{8}$ & $-1.3070(0)$ & $0.9788(1)$ & $-0.9540(2)$ & $1.0515(0)$ & $-0.2307(2)$ \\
\hline \hline
\end{tabular}




$$
P_{e e}^{+}(z, 0)=\frac{1+z^{2}}{(1-z)_{+}}+\frac{3}{2} \delta(1-z)
$$

being the regularized Altarelli-Parisi splitting function. The one-loop structure function is used here to ensure the cancellation of collinear singularities, instead of using the most commonly used resummed ones. The cancellation is shown in Table VI. We vary the mass of the electron with a factor of $k$ from $2^{-4}$ to $2^{8}$ and find the result unchanged. Also, we can see that singular terms only appear in $\sigma_{V+S}$ and $\sigma_{\mathrm{SC}}$ parts.

\section{APPENDIX B: TRIPLE HIGGS COUPLINGS IN 2HDM}

The Higgs potential of $2 \mathrm{HDM}$ determines the selfcouplings among Higgs bosons. Among them, the triple Higgs couplings (THC) can be parametrized as a function of the 2HDM parameters $m_{h^{0}}, m_{H^{0}}, m_{A^{0}}, m_{H^{ \pm}}, \tan \beta, \alpha$, and $\lambda_{5}$. At tree level, these couplings are independent of the Yukawa types, and they are given as follows:

$$
\begin{aligned}
& \lambda_{h^{0} h^{0} h^{0}}^{2 \mathrm{HDM}}=\frac{1}{v}\left\{-\frac{3}{s_{2 \beta}^{2}}\left[\left(2 c_{\alpha+\beta}+s_{2 \alpha} s_{\beta-\alpha}\right) s_{2 \beta} m_{h^{0}}^{2}-4 c_{\beta-\alpha}^{2} c_{\beta+\alpha} m_{12}^{2}\right]\right\}, \\
& \lambda_{H^{0} h^{0} h^{0}}^{2 \mathrm{HDM}}=\frac{1}{v}\left\{-\frac{c_{\beta-\alpha}}{s_{2 \beta}^{2}}\left[\left(2 m_{h^{0}}^{2}+m_{H^{0}}^{2}\right) s_{2 \alpha} s_{2 \beta}-2\left(3 s_{2 \alpha}-s_{2 \beta}\right) m_{12}^{2}\right]\right\}, \\
& \lambda_{h^{0} H^{0} H^{0}}^{2 \mathrm{HDM}}=\frac{1}{v}\left\{\frac{s_{\beta-\alpha}}{s_{2 \beta}^{2}}\left[\left(m_{h^{0}}^{2}+2 m_{H^{0}}^{2}\right) s_{2 \alpha} s_{2 \beta}-2\left(3 s_{2 \alpha}+s_{2 \beta}\right) m_{12}^{2}\right]\right\}, \\
& \lambda_{h^{0} H^{ \pm} H^{\mp}}^{2 \mathrm{HDM}}=\frac{1}{v}\left\{\frac{1}{s_{2 \beta}^{2}}\left[\left(m_{h^{0}}^{2}-2 m_{H^{ \pm}}^{2}\right) s_{\beta-\alpha} s_{2 \beta}^{2}-2 c_{\beta+\alpha}\left(m_{h^{0}}^{2} s_{2 \beta}-2 m_{12}^{2}\right)\right]\right\}, \\
& \lambda_{h^{0} A^{0} A^{0}}^{2 \mathrm{HDM}}= \frac{1}{v}\left\{\frac{1}{s_{2 \beta}^{2}}\left[\left(m_{h^{0}}^{2}-2 m_{A^{0}}^{2}\right) s_{\beta-\alpha} s_{2 \beta}^{2}-2 c_{\beta+\alpha}\left(m_{h^{0}}^{2} s_{2 \beta}-2 m_{12}^{2}\right)\right]\right\}, \\
& \lambda_{H^{0} H^{0} H^{0}}^{2 \mathrm{HDM}}=\frac{1}{v}\left\{-\frac{3}{s_{2 \beta}^{2}}\left[\left(2 s_{\alpha+\beta}-s_{2 \alpha} c_{\beta-\alpha}\right) s_{2 \beta} m_{H^{0}}^{2}-4 s_{\beta-\alpha}^{2} s_{\alpha+\beta} m_{12}^{2}\right]\right\}, \\
& \lambda_{H^{0} A^{0} A^{0}}^{2 \mathrm{HDM}}=\frac{1}{v}\left\{-\frac{1}{s_{2 \beta}^{2}}\left[s_{\alpha+\beta}\left(2 m_{H^{0}}^{2} s_{2 \beta}-4 m_{12}^{2}\right)-\left(m_{H^{0}}^{2}-2 m_{A^{0}}^{2}\right) s_{2 \beta}^{2} c_{\beta-\alpha}\right]\right\}, \\
& \lambda_{H^{ \pm} H^{\mp} H^{0}}^{2 \mathrm{HDM}}=\frac{1}{v}\left\{-\frac{1}{s_{2 \beta}^{2}}\left[s_{\alpha+\beta}\left(2 m_{H^{0}}^{2} s_{2 \beta}-4 m_{12}^{2}\right)-\left(m_{H^{0}}^{2}-2 m_{H^{ \pm}}^{2}\right) s_{2 \beta}^{2} c_{\beta-\alpha}\right]\right\},
\end{aligned}
$$

where $v$ is the VEV and $m_{12}^{2}$ can be derived from $m_{A}, \beta, \lambda_{5}$, and $v$, according to the relation given in Eq. (22). We have used the notations $s_{\theta}$ and $c_{\theta}$ as shorthand notations for $\sin (\theta)$ and $\cos (\theta)$, respectively. The mixing angle $\beta$ is defined via $\tan \beta=v_{2} / v_{1}$.

As mentioned in the second paragraph of Sec. IV, two different physical parametrizations have been used in this paper, which yields different values of $v$, and hence different THCs. Besides the overall factor $1 / v$, the remaining terms of Eqs. (B1)-(B8) still depend on $v$, due to the fact that $m_{12}^{2}$ is obtained via Eq. (22) and terms proportional to $\lambda_{5} v^{2}$ will appear. In order to further investigate the dependence, we rewrite the THCs as

$$
\lambda_{i} \equiv \frac{(246.220 \mathrm{GeV})^{2}}{v} \hat{\lambda}_{i}, \quad i=h^{0} h^{0} h^{0}, H^{0} h^{0} h^{0}, \ldots,
$$

where the dimensionless couplings $\hat{\lambda}_{i}$ are just the terms in the curly brackets of Eqs. (B1)-(B8) divided by $(246.220 \mathrm{GeV})^{2}$.

In Table VII, all the $\hat{\lambda}_{i}$ values in the two cases are tabulated, as well as corresponding values of $m_{12}^{2}$. It can be seen from the table that in the case of BP2-h (BP2-H), some of the THCs are quite large compared with $h^{0} h^{0} h^{0}\left(H^{0} H^{0} H^{0}\right)$. Although these THCs are sizeable, we have checked that they still respect the 
TABLE VII. Dimensionless couplings $\hat{\lambda}_{i}$ with two typical values of $v$; the inputs of each of the benchmark points are given in Table II. $\hat{\lambda}_{i}$ values are defined from the THCs via Eq. (B9). The values of $v$ are taken as explained in the second paragraph of Sec. IV.

\begin{tabular}{lcrrrrrrrrr}
\hline \hline$v(\mathrm{GeV})$ & BPs & $\hat{\lambda}_{h^{0} h^{0} h^{0}}$ & $\hat{\lambda}_{H^{0} h^{0} h^{0}}$ & $\hat{\lambda}_{h^{0} H^{0} H^{0}}$ & $\hat{\lambda}_{h^{0} H^{ \pm} H^{ \pm}}$ & $\hat{\lambda}_{h^{0} A^{0} A^{0}}$ & $\hat{\lambda}_{H^{0} H^{ \pm} H^{ \pm}}$ & $\hat{\lambda}_{H^{0} A^{0} A^{0}}$ & $\hat{\lambda}_{H^{0} H^{0} H^{0}}$ & $m_{12}^{2}\left(\mathrm{GeV}^{2}\right)$ \\
\hline \multirow{2}{2}{246.220} & BP1-h & -0.763 & -0.048 & -0.810 & -0.375 & 0.353 & -0.030 & 0.028 & -0.195 & 3126.00 \\
& BP2-h & -0.773 & -0.049 & -3.257 & -3.199 & -0.285 & -2.305 & -2.292 & -6.917 & 104480.72 \\
& BP1-H & 0.025 & -0.240 & -0.002 & 0.048 & 0.047 & -0.895 & -0.884 & -0.774 & 2839.78 \\
& BP2-H & -0.036 & -0.235 & -0.001 & 0.335 & 0.335 & -11.807 & -11.807 & -0.773 & 3567.16 \\
& BP1-h & -0.763 & -0.047 & -0.789 & -0.388 & 0.340 & -0.238 & -0.179 & -0.814 & 3064.92 \\
& BP2-h & -0.773 & -0.049 & -3.257 & -3.199 & -0.285 & -2.305 & -2.292 & -6.917 & 104480.72 \\
& BP1-H & -0.037 & -0.228 & -0.0003 & 0.027 & 0.026 & -0.880 & -0.869 & -0.774 & 2992.36 \\
& BP2-H & -0.793 & 0.030 & 0.016 & 0.083 & 0.083 & -11.527 & -11.527 & -0.773 & 6981.21 \\
\hline \hline
\end{tabular}

conditions of perturbativity and the unitarity of scattering amplitudes of Higgs bosons in the model, as listed in Sec. II.

On the other hand, although the deviation between the two VEVs is smaller than $1.5 \%$, it is observed that the values of $\hat{\lambda}_{i}$ can be significantly different, such as $\hat{\lambda}_{H^{0} H^{ \pm} H^{ \pm}}$, $\hat{\lambda}_{H^{0} A^{0} A^{0}}$, and $\hat{\lambda}_{H^{0} H^{0} H^{0}}$ in the case of BP1-h, and $\hat{\lambda}_{h^{0} h^{0} h^{0}}$, $\hat{\lambda}_{H^{0} h^{0} h^{0}}, \hat{\lambda}_{h^{0} H^{ \pm} H^{ \pm}}$, and $\hat{\lambda}_{h^{0} A^{0} A^{0}}$ in the case of BP2-H, which indicates that the corresponding THCs are very sensitive to the value of $v$. As $v$ only appears in $\hat{\lambda}_{i}$ in terms of $\lambda_{5} v^{2}$, this also means that those THCs are very sensitive to the value of $\lambda_{5}$ (a deviation smaller than $3 \%$ will change the corresponding $\hat{\lambda}_{i}$ drastically). Usually, the effects caused by the different values of $v$ are regarded as higher-order corrections. But from Table VII, we can see that in some cases this will lead to totally different theoretical predictions; e.g., the decay width of $H^{0} \rightarrow A^{0} A^{0}$ in the case of BP1-h. Fortunately, in this work, this difference in $\hat{\lambda}_{i}$ does not change our results very much (see also the discussion below).

In Fig. 15, we show the dependence of $\hat{\lambda}_{i}$ upon the parameter $\lambda_{5}$ for each of the benchmark mass spectra and fixed parameters $\alpha$ and $\tan \beta$ given in Table II, where $v=243.137 \mathrm{GeV}$ is used. In the benchmark points BP1$\mathrm{h}(\mathrm{H})$ and $\mathrm{BP} 2-\mathrm{h}(\mathrm{H})$, where $h^{0}\left(H^{0}\right)$ is assumed to be the SM-like Higgs boson, the $\hat{\lambda}_{i}$ 's related to $h^{0}\left(H^{0}\right)$ are depicted as solid lines, while the others are depicted by dashed lines, as they can also contribute via the renormalization of wave functions or mixing angles. We have merged several pairs of lines in the figure, since they are identical or very close to each other, which can be easily learned from Eqs. (B1)-(B8) and the mass spectra in Table II.

The range of $\lambda_{5}$ between two vertical dashed lines denotes the allowed region for each benchmark point. It is observed from the figure that the gradients of some lines are quite large; thus, the corresponding $\hat{\lambda}_{i}$ 's change drastically in the allowed range of $\lambda_{5}$, such as $\hat{\lambda}_{h^{0} h^{0} h^{0}}$ in the case of BP1-H, which changes from 7.5 to -0.05 . As we discussed before, the dependence of $\hat{\lambda}_{i}$ on $v$ can be converted into the dependence on $\lambda_{5}$. This explains the discrepancy of some $\hat{\lambda}_{i}$ values caused by the different values of $v$ in Table VII. Also, it can be estimated that the effect caused by different values of $v$ (after removing the overall factor) is much smaller than the variation of $\lambda_{5}$ in the allowed range, since the difference between the values of $v$ can be taken as a deviation smaller than $3 \%$ on $\lambda_{5}$.

We have discussed the dependence of THCs on $v$. In fact, the allowed ranges of $\lambda_{5}$ also depend on it. In Table VIII, we show the allowed ranges of $\lambda_{5}$ for all the BPs. As mentioned in Sec. IV, we determine the allowed ranges of $\lambda_{5}$ by fixing other parameters and using the constraints from vacuum stability, unitarity, and perturbativity. In this procedure we have taken $v=246.220 \mathrm{GeV}$, the same as the value used in the scan of the 2HDM parameter space. However, in the calculation of production processes, $v=243.137 \mathrm{GeV}$ is used.

It can be observed from Table VIII that both the upper and lower bounds of $\lambda_{5}$ will change when a different $v$ is used. The largest deviation is found in the lower bound of BP2-h, which is about $10 \%$. This cannot be simply explained by an overall factor related to $v$ only, and needs further investigation.

The constraints used to determine the ranges of $\lambda_{5}$ can be expressed as several dimensionless inequalities involving $v$ and the 2HDM parameters: $m_{h^{0}}, m_{H^{0}}$, $m_{A^{0}}, m_{H^{ \pm}}, \tan \beta, \alpha$, and $\lambda_{5}$. Besides an overall factor $1 / v^{2}$, the only dependence on $v$ in these inequalities is the term $\lambda_{5} v^{2}$, similar to the case of THCs. This means we can remove part of the effects from $v$ by looking into $\lambda_{5} v^{2}$ or $m_{12}^{2}$. In Table VIII, the ranges of $\lambda_{5} v^{2}$ and $m_{12}^{2}$ are also presented. It is found that the upper bounds of $\lambda_{5} v^{2}$ and $m_{12}^{2}$ are almost the same even when different values of $v$ are used, while the lower bounds are quite different. We have checked the constraints related to each bound and found that all the upper bounds are determined by the constraints from vacuum stability, 


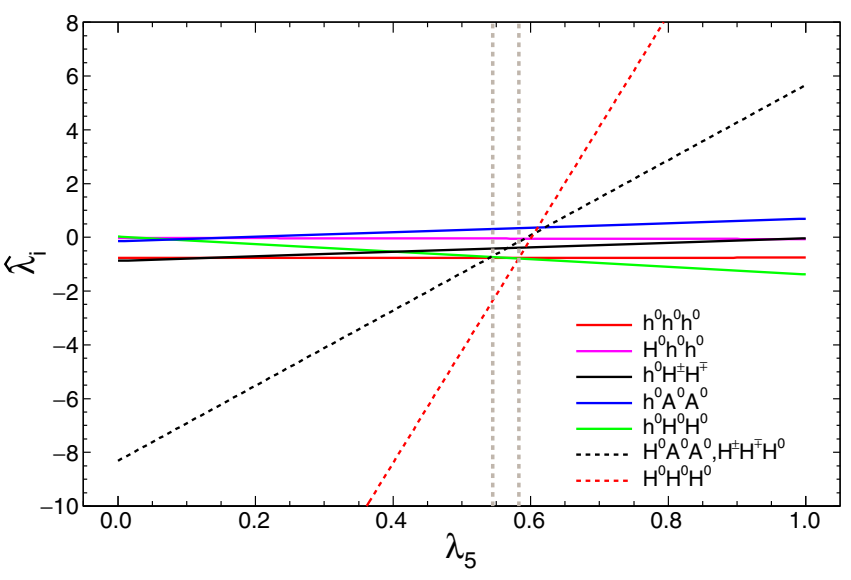

(a) BP1-h

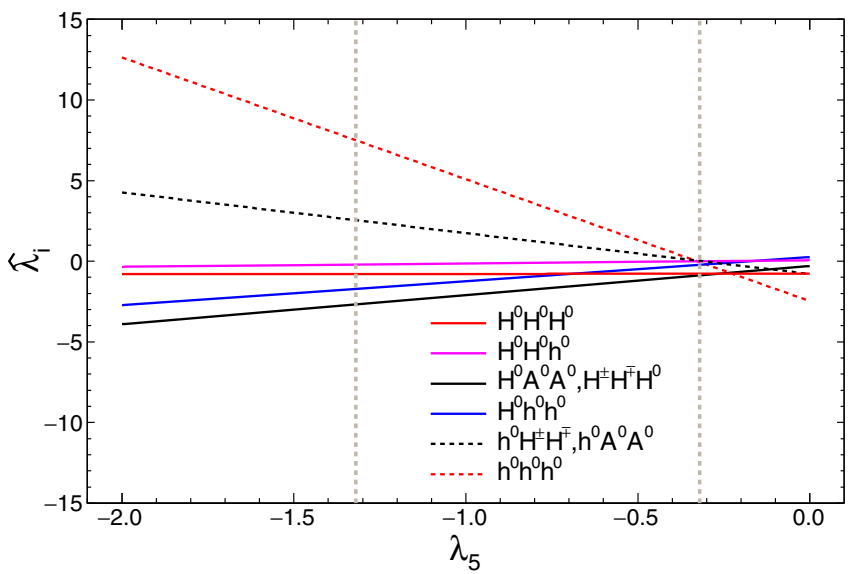

(c)BP1-H

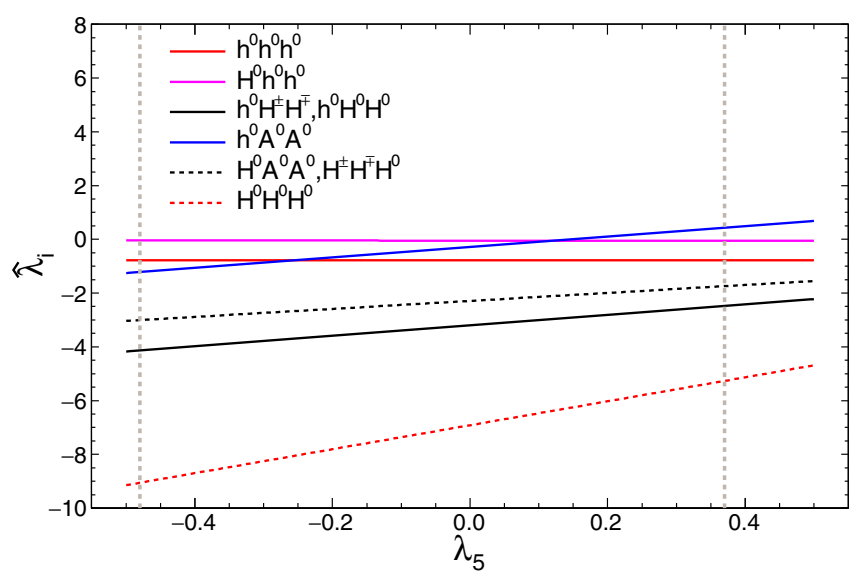

(b) BP2-h

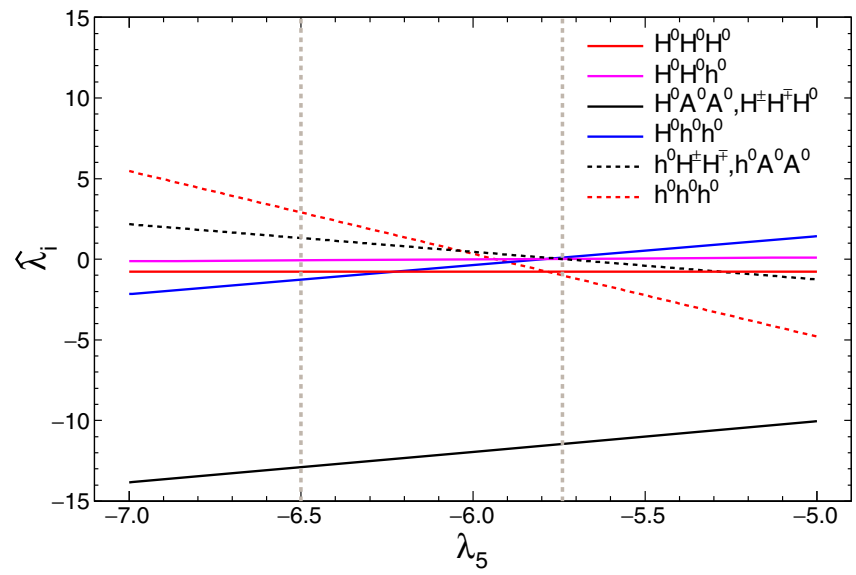

(d) BP2-H

FIG. 15. The dependence of $\hat{\lambda}_{i}$ upon $\lambda_{5}$ for each of the benchmark points is demonstrated. In each plot, the range between the two vertical dashed lines corresponds to the allowed $\lambda_{5}$ by theoretical and experimental constraints and bounds. $v$ is taken to be $243.137 \mathrm{GeV}$.

while all the lower bounds are determined by the constraints from unitarity. The constraints from vacuum stability are homogeneous, so they are not affected by the choice of $v$. On the other hand, the constraints from unitarity are inhomogeneous, and some of them are even nonlinear. This leads to more complicated $v$ dependence of $\lambda_{5} v^{2}$ and $m_{12}^{2}$. From the table, we can see that the effects of different $v$ 's on the lower bounds can be negligible, such as $m_{12}^{2}$ in BP1-h, but they can also be large, such as $m_{12}^{2}$ in BP2-H.

From the above analysis, we can conclude that deviations of the upper bounds of $\lambda_{5}$ can be easily understood by multiplying the ratio of two different $v^{2}$ values, while the lower bounds are more complicated. Moreover, together

TABLE VIII. Allowed ranges of $\lambda_{5}, \lambda_{5} v^{2}$, and $m_{12}^{2}$ with two typical values of $v$. The constraints from vacuum stability, unitarity, and perturbativity are used to determine the ranges. The values of $v$ are taken as explained in the second paragraph of Sec. IV.

\begin{tabular}{lccccc}
\hline \hline$v(\mathrm{GeV})$ & BPs & BP1-h & BP2-h & BP1-H & BP2-H \\
\hline 246.220 & $\lambda_{5}$ & {$[0.545,0.583]$} & {$[-0.482,0.377]$} & {$[-1.330,-0.294]$} & {$[-6.500,-5.737]$} \\
& $\lambda_{5} v^{2}\left(\mathrm{GeV}^{2}\right)$ & {$[33040,35368]$} & {$[-29197,22874]$} & {$[-80612,-17848]$} & {$[-394063,-347807]$} \\
& $m_{12}^{2}\left(\mathrm{GeV}^{2}\right)$ & {$[2970,3132]$} & {$[92844,113597]$} & {$[-16351,3365]$} & {$[-12986,4649]$} \\
243.137 & $\lambda_{5}$ & {$[0.560,0.598]$} & {$[-0.441,0.387]$} & {$[-1.337,-0.302]$} & {$[-6.601,-5.884]$} \\
& $\lambda_{5} v^{2}\left(\mathrm{GeV}^{2}\right)$ & {$[33099,35367]$} & {$[-26094,22872]$} & {$[-79032,-17847]$} & {$[-390217,-347807]$} \\
& $m_{12}^{2}\left(\mathrm{GeV}^{2}\right)$ & {$[2974,3132]$} & {$[94081,113597]$} & {$[-15855,3366]$} & {$[-11520,4649]$} \\
\hline \hline
\end{tabular}


with the situation of THCs, this hints that it is better to choose independent physical parameters with the same mass dimension.

\section{APPENDIX C: ON-SHELL PINCHED TADPOLE SCHEME IN THE 2HDM}

In this Appendix, we present more details about the onshell PTS $[104,105]$, which is used in the calculation of the production $e^{+} e^{-} \rightarrow Z \phi$. For the study on $\phi \rightarrow f \bar{f}$ decay, as it is a work following Ref. [38], the same renormalization scheme as in Ref. [38] is used and will not be discussed here.

\section{Definition of renormalization constants}

In the following, we introduce renormalized quantities and renormalization constants in the on-shell PTS, where an index 0 is used to label bare quantities.

1. Gauge and fermion sectors: Things are similar to the SM for these two sectors. The renormalization of gauge boson and fermion masses are given by

$$
\begin{aligned}
& m_{V, 0}^{2}=m_{V}^{2}+\delta m_{V}^{2}, \quad V=W, Z, \\
& m_{f, 0}=m_{f}+\delta m_{f},
\end{aligned}
$$

and the electric charge by

$$
e_{0}=\left(1+\delta Z_{e}\right) e .
$$

Meanwhile, the renormalization of the gauge fields are defined as

$$
\begin{aligned}
W_{0}^{ \pm} & =\left(1+\frac{1}{2} \delta Z_{W W}\right) W^{ \pm} \\
\left(\begin{array}{l}
Z \\
A
\end{array}\right)_{0} & =\left(\begin{array}{cc}
1+\frac{1}{2} \delta Z_{Z Z} & \frac{1}{2} \delta Z_{Z A} \\
\frac{1}{2} \delta Z_{A Z} & 1+\frac{1}{2} \delta Z_{A A}
\end{array}\right)\left(\begin{array}{l}
Z \\
A
\end{array}\right),
\end{aligned}
$$

while for the fermion field, due to its left- and righthanded chirality, it has independent field renormalization constants:

$$
\begin{aligned}
f_{0}^{L} & =\left(1+\frac{1}{2} \delta Z_{f}^{L}\right) f^{L} \\
f_{0}^{R} & =\left(1+\frac{1}{2} \delta Z_{f}^{R}\right) f^{R}
\end{aligned}
$$

2. Higgs sector: The renormalization is performed in a Higgs physics basis. The renormalization of Higgs masses is defined as

$$
m_{\phi, 0}^{2}=m_{\phi}^{2}+\delta m_{\phi}^{2}, \quad \phi=G^{ \pm}, H^{ \pm}, G^{0}, H^{0}, h^{0},
$$

while the renormalization of Higgs fields is given by

$$
\begin{aligned}
& \left(\begin{array}{c}
H^{0} \\
h^{0}
\end{array}\right)_{0}=\left(\begin{array}{cc}
1+\frac{1}{2} \delta Z_{H^{0} H^{0}} & \frac{1}{2} \delta Z_{H^{0} h^{0}} \\
\frac{1}{2} \delta Z_{h^{0} H^{0}} & 1+\frac{1}{2} \delta Z_{h^{0} h^{0}}
\end{array}\right)\left(\begin{array}{c}
H^{0} \\
h^{0}
\end{array}\right), \\
& \left(\begin{array}{c}
G^{0} \\
A^{0}
\end{array}\right)_{0}=\left(\begin{array}{cc}
1+\frac{1}{2} \delta Z_{G^{0} G^{0}} & \frac{1}{2} \delta Z_{G^{0} A^{0}} \\
\frac{1}{2} \delta Z_{A^{0} G^{0}} & 1+\frac{1}{2} \delta Z_{A^{0} A^{0}}
\end{array}\right)\left(\begin{array}{c}
G^{0} \\
A^{0}
\end{array}\right), \\
& \left(\begin{array}{c}
G^{ \pm} \\
H^{ \pm}
\end{array}\right)_{0}=\left(\begin{array}{cc}
1+\frac{1}{2} \delta Z_{G^{ \pm} G^{ \pm}} & \frac{1}{2} \delta Z_{G^{ \pm} H^{ \pm}} \\
\frac{1}{2} \delta Z_{H^{ \pm} G^{ \pm}} & 1+\frac{1}{2} \delta Z_{H^{ \pm} H^{ \pm}}
\end{array}\right)\left(\begin{array}{c}
G^{ \pm} \\
H^{ \pm}
\end{array}\right) .
\end{aligned}
$$

For the mixing angles, they are defined as

$$
\begin{aligned}
& \alpha_{0}=\alpha+\delta \alpha, \\
& \beta_{0}=\beta+\delta \beta .
\end{aligned}
$$

We have skipped the renormalization of $\lambda_{5}$, as it is not needed in our calculation. In order to expose the difference between the on-shell PTS and the other tadpole schemes, we also provide the renormalization of the tadpoles in the standard tadpole scheme, which is defined as given below:

$$
\begin{aligned}
T_{H^{0}, 0} & =T_{H^{0}}+\delta T_{H^{0}}, \\
T_{h^{0}, 0} & =T_{h^{0}}+\delta T_{h^{0}} .
\end{aligned}
$$

In contrast, the on-shell PTS is based on the FJ tadpole scheme [32], where the shifts of the VEVs $v_{1,2} \rightarrow v_{1,2}+\Delta v_{1,2}$ are introduced. We can choose the values of $\Delta v_{1,2}$ order by order to make renormalized tadpoles vanishing in the perturbation expansion. In such a procedure, we are able to appropriately allocate the tadpole contributions and define all counterterms in a gauge-independent way.

It is noteworthy that in the on-shell PTS, there is no need to define the tadpole counterterms given in Eq. (C8) (i.e., $\delta T_{H^{0}, h^{0}}$ ) anymore. Instead, their roles are replaced by $\Delta T_{H^{0}, h^{0}}$, which are caused by the shift of the VEVs and are given in Refs. $[104,105]$ as

$$
\begin{aligned}
\Delta T_{H^{0}} & =\left(c_{\alpha} \Delta v_{1}+s_{\alpha} \Delta v_{2}\right) m_{H^{0}}^{2}, \\
\Delta T_{h^{0}} & =\left(-s_{\alpha} \Delta v_{1}+c_{\alpha} \Delta v_{2}\right) m_{h^{0}}^{2} .
\end{aligned}
$$

To avoid confusion, we have deliberately changed the convention of $\delta T$ in Refs. [104,105] to $\Delta T$ in order to indicate that the origin and meaning of $\Delta T$ are different from $\delta T$ given in Eq. (C8). Such a convention has also been adopted in Ref. [101]. 


\section{Renormalization conditions}

$\Delta v_{1,2} / \Delta T_{H^{0}, h^{0}}$ will appear in self-energies as well as vertices. It has already been revealed in Refs. $[101,104]$ that the effect of $\Delta v_{1,2} / \Delta T_{H^{0}, h^{0}}$ can be easily included by adding possible tadpole diagrams to generic one-particle irreducible diagrams. In this scheme, well-known on-shell conditions are used everywhere. The differences between the wellknown on-shell scheme and this scheme are as follows:

1. Tadpole counterterms $\delta T_{H^{0}, h^{0}}$ defined in Eq. (C8) vanish in this scheme.

2. Self-energies $\Sigma\left(k^{2}\right)$ are replaced with $\Sigma^{\operatorname{tad}}\left(k^{2}\right)$ by adding possible tadpole contributions.

It should be pointed out that the derivatives of self-energies $\Sigma^{\prime}\left(k^{2}\right)$ remain unchanged due to the fact that tadpole contributions are independent of external momentum $k$; thus, most wave function renormalization constants are the same.

In the following, we present more necessary information about this on-shell PTS. For more details, please refer to Ref. [104].

In gauge and fermion sectors, the same on-shell conditions as in the SM are used [94]. Among all the renormalization constants in these sectors, three are different: $\delta m_{W}^{2}, \delta m_{Z}^{2}$, and $\delta m_{f}$. Only the first two are used in this work; they are given by

$$
\delta m_{V}^{2}=\operatorname{Re} \Sigma_{V V}^{\mathrm{tad}, T}\left(m_{V}^{2}\right), \quad V=W, Z .
$$

In the Higgs sector, the renormalized self-energy of the Higgs field $\phi$ is the following finite combination of the unrenormalized self-energy:

$$
\begin{aligned}
\hat{\Sigma}_{\phi \phi}\left(k^{2}\right) & =\Sigma_{\phi \phi}^{\mathrm{tad}}\left(k^{2}\right)-\delta m_{\phi}^{2}+\left(k^{2}-m_{\phi}^{2}\right) \delta Z_{\phi \phi}, \\
\phi & =G^{ \pm}, H^{ \pm}, G^{0}, A^{0}, H^{0}, h^{0},
\end{aligned}
$$

while for the mixing one, it is

$$
\begin{aligned}
\hat{\Sigma}_{\phi_{1} \phi_{2}}\left(k^{2}\right)= & \Sigma_{\phi_{1} \phi_{2}}^{\mathrm{tad}}\left(k^{2}\right)+\frac{1}{2} \delta Z_{\phi_{1} \phi_{2}}\left(k^{2}-m_{\phi_{1}}^{2}\right) \\
& +\frac{1}{2} \delta Z_{\phi_{2} \phi_{1}}\left(k^{2}-m_{\phi_{2}}^{2}\right),
\end{aligned}
$$

with $\left(\phi_{1}, \phi_{2}\right)=\left(G^{ \pm}, H^{ \pm}\right),\left(G^{0}, A^{0}\right)$, and $\left(H^{0}, h^{0}\right)$.

The renormalization conditions for the masses and wave functions in Higgs sector are given as follows:

1. For the masses, on-shell conditions are used:

$$
\operatorname{Re} \hat{\Sigma}_{\phi \phi}\left(m_{\phi}^{2}\right)=0, \quad \phi=H^{ \pm}, A^{0}, H^{0}, h^{0} .
$$

These conditions ensure that the physical masses of Higgs bosons are pole masses of corresponding renormalized propagators, from which we obtain

$$
\delta m_{\phi}^{2}=\operatorname{Re} \Sigma_{\phi \phi}^{\mathrm{tad}}\left(m_{\phi}^{2}\right), \quad \phi=H^{ \pm}, A^{0}, H^{0}, h^{0} .
$$

2. For the wave functions, we require the residue of the renormalized propagator of $\phi$ to be one:

$$
\begin{aligned}
& \left.\operatorname{Re} \hat{\Sigma}_{\phi \phi}^{\prime}\left(k^{2}\right)\right|_{k^{2}=m_{\phi}^{2}}=0, \\
& \phi=G^{ \pm}, H^{ \pm}, G^{0}, A^{0}, H^{0}, h^{0},
\end{aligned}
$$

and the mixing self-energies vanish when one of the external particles is on shell:

$$
\begin{aligned}
& \hat{\Sigma}_{\phi_{1} \phi_{2}}\left(m_{\phi_{1}}^{2}\right)=\hat{\Sigma}_{\phi_{1} \phi_{2}}\left(m_{\phi_{2}}^{2}\right)=0, \\
& \left(\phi_{1}, \phi_{2}\right)=\left(G^{ \pm}, H^{ \pm}\right),\left(G^{0}, A^{0}\right),\left(H^{0}, h^{0}\right) .
\end{aligned}
$$

From the equations, we obtain

$$
\begin{aligned}
\delta Z_{\phi \phi} & =-\operatorname{Re} \Sigma_{\phi \phi}^{\prime}\left(m_{\phi \phi}^{2}\right), \\
\phi & =G^{ \pm}, H^{ \pm}, G^{0}, A^{0}, H^{0}, h^{0}, \\
\delta Z_{\phi_{1} \phi_{2}} & =\frac{2 \operatorname{Re} \Sigma_{\phi_{1} \phi_{2}}^{\mathrm{tad}}\left(m_{\phi_{2}}^{2}\right)}{m_{\phi_{1}}^{2}-m_{\phi_{2}}^{2}}, \\
\left(\phi_{1}, \phi_{2}\right) & =\left(G^{ \pm}, H^{ \pm}\right),\left(G^{0}, A^{0}\right),\left(H^{0}, h^{0}\right) .
\end{aligned}
$$

The renormalization of the mixing angles might be the most important part of this Appendix. As the FJ tadpole scheme is applied, gauge-independent definitions for the counterterms $\delta \alpha$ and $\delta \beta$ are possible. In Ref. [101], $\overline{\mathrm{MS}}$ subtraction is used, while in this work, we follow the procedure in Ref. [104] where the pinch technique (see, e.g., Ref. [106]) is applied:

1. First, the pinched self-energies $\bar{\Sigma}$ are obtained with the help of the pinch technique. It is found to be a sum of two parts:

$$
\bar{\Sigma}\left(k^{2}\right)=\left.\Sigma^{\mathrm{tad}}\right|_{\xi=1}\left(k^{2}\right)+\Sigma^{\mathrm{add}}\left(k^{2}\right),
$$

where $\xi$ stands for the gauge-fixing parameters $\xi_{Z}$, $\xi_{W}$, and $\xi_{\gamma}$ of the $R_{\xi}$ gauge. As shown in Eq. (C16), the first part has the same form as the tadpole selfenergies evaluated in the Feynman gauge, and the second one is an additional contribution which is explicitly independent of the gauge fixing parameter $\xi$.

2. The counterterms are then defined through the above pinched self-energies with the on-shell scale, namely

$$
\begin{aligned}
& \delta \alpha=\frac{\operatorname{Re} \bar{\Sigma}_{H^{0} h^{0}}\left(m_{H^{0}}^{2}\right)+\operatorname{Re} \bar{\Sigma}_{H^{0} h^{0}}\left(m_{h^{0}}^{2}\right)}{2\left(m_{H^{0}}^{2}-m_{h^{0}}^{2}\right)}, \\
& \delta \beta=-\frac{\operatorname{Re} \bar{\Sigma}_{G^{0} A^{0}}(0)+\operatorname{Re} \bar{\Sigma}_{G^{0} A^{0}}\left(m_{A^{0}}^{2}\right)}{2 m_{A^{0}}^{2}},
\end{aligned}
$$

where the definition for $\delta \beta$ from the $C P$-odd Higgs sector has been chosen. 
More details about this on-shell PTS can be found in Ref. [104]. For completeness, we list here the additional parts in the pinched self-energies as the end of this Appendix:

$$
\begin{aligned}
\Sigma_{H^{0} h^{0}}^{\text {add }}\left(k^{2}\right)= & \frac{g^{2} s_{\beta-\alpha} c_{\beta-\alpha}}{32 \pi^{2} c_{W}^{2}}\left(k^{2}-\frac{m_{H^{0}}^{2}+m_{h^{0}}^{2}}{2}\right)\left\{\left[B_{0}\left(k^{2} ; m_{Z}^{2}, m_{A^{0}}^{2}\right)-B_{0}\left(k^{2} ; m_{Z}^{2}, m_{Z}^{2}\right)\right]\right. \\
& \left.+2 c_{W}^{2}\left[B_{0}\left(k^{2} ; m_{W}^{2}, m_{H^{ \pm}}^{2}\right)-B_{0}\left(k^{2} ; m_{W}^{2}, m_{W}^{2}\right)\right]\right\}, \\
\Sigma_{G^{0} A^{0}}^{\text {add }}\left(k^{2}\right)= & \frac{g^{2} s_{\beta-\alpha} c_{\beta-\alpha}}{32 \pi^{2} c_{W}^{2}}\left(k^{2}-\frac{m_{A^{0}}^{2}}{2}\right)\left[B_{0}\left(k^{2} ; m_{Z}^{2}, m_{H^{0}}^{2}\right)-B_{0}\left(k^{2} ; m_{Z}^{2}, m_{h^{0}}^{2}\right)\right],
\end{aligned}
$$

where $B_{0}$ is the scalar two-point function [138].

[1] S. Chatrchyan et al. (CMS Collaboration), Phys. Lett. B 716, 30 (2012).

[2] G. Aad et al. (ATLAS Collaboration), Phys. Lett. B 716, 1 (2012).

[3] G. Aad et al. (ATLAS, CMS Collaboration), Phys. Rev. Lett. 114, 191803 (2015).

[4] G. Aad et al. (ATLAS, CMS Collaboration), J. High Energy Phys. 08 (2016) 045.

[5] M. Aaboud et al. (ATLAS Collaboration), Phys. Rev. D 98, 052003 (2018).

[6] A. M. Sirunyan et al. (CMS Collaboration), Phys. Rev. Lett. 121, 121801 (2018).

[7] M. Aaboud et al. (ATLAS Collaboration), Phys. Rev. D 99, 072001 (2019).

[8] A. M. Sirunyan et al. (CMS Collaboration), Phys. Lett. B 779, 283 (2018).

[9] A. M. Sirunyan et al. (CMS Collaboration), Phys. Rev. Lett. 120, 231801 (2018).

[10] M. Aaboud et al. (ATLAS Collaboration), Phys. Lett. B 784, 173 (2018).

[11] A. M. Sirunyan et al. (CMS Collaboration), Eur. Phys. J. C 79, 421 (2019).

[12] G. Aad et al. (ATLAS Collaboration), Phys. Rev. D 101, 012002 (2020).

[13] CMS Collaboration, Report No. CMS-PAS-FTR-18-011, 2018.

[14] P. Garcia-Abia and W. Lohmann, Eur. Phys. J. Direct 2, 1 (2000).

[15] M. Dong et al. (CEPC Study Group), arXiv:1811.10545.

[16] D. M. Asner et al., in Proceedings, 2013 Community Summer Study on the Future of U.S. Particle Physics: Snowmass on the Mississippi (CSS2013): Minneapolis, MN, USA, 2013 (2013) [arXiv:1310.0763].

[17] M. E. Peskin, arXiv:1207.2516.

[18] A. Blondel, A. Chao, W. Chou, J. Gao, D. Schulte, and K. Yokoya, in ICFA Beam Dynamics Workshop on Accelerators for a Higgs Factory: Linear v. Circular (HF2012) Batavia, IL, 2012 (2013) [arXiv:1302.3318].

[19] R. Belusevic and T. Higo, arXiv:1208.4956.
[20] H. Baer, T. Barklow, K. Fujii, Y. Gao, A. Hoang, S. Kanemura, J. List, H.E. Logan, A. Nomerotski, M. Perelstein et al., arXiv:1306.6352.

[21] G. Moortgat-Pick et al., Phys. Rep. 460, 131 (2008).

[22] A. Arbey et al., Eur. Phys. J. C 75, 371 (2015).

[23] D. Lopez-Val, J. Sola, and N. Bernal, Phys. Rev. D 81, 113005 (2010).

[24] J. Fleischer and F. Jegerlehner, Nucl. Phys. B216, 469 (1983).

[25] B. A. Kniehl, Z. Phys. C 55, 605 (1992).

[26] A. Denner, J. Kublbeck, R. Mertig, and M. Bohm, Z. Phys. C 56, 261 (1992).

[27] https://minami-home.kek.jp

[28] G. Belanger, F. Boudjema, J. Fujimoto, T. Ishikawa, T. Kaneko, K. Kato, and Y. Shimizu, Phys. Lett. B 559, 252 (2003).

[29] N. Sakai, Phys. Rev. D 22, 2220 (1980).

[30] T. Inami and T. Kubota, Nucl. Phys. B179, 171 (1981).

[31] M. Drees and K.-i. Hikasa, Phys. Rev. D 41, 1547 (1990).

[32] J. Fleischer and F. Jegerlehner, Phys. Rev. D 23, 2001 (1981).

[33] B. A. Kniehl, Nucl. Phys. B376, 3 (1992).

[34] A. Dabelstein, Nucl. Phys. B456, 25 (1995).

[35] H. E. Haber, M. J. Herrero, H. E. Logan, S. Penaranda, S. Rigolin, and D. Temes, Phys. Rev. D 63, 055004 (2001).

[36] J. Guasch, W. Hollik, and S. Penaranda, Phys. Lett. B 515, 367 (2001).

[37] S. Kanemura, M. Kikuchi, and K. Yagyu, Phys. Lett. B 731, 27 (2014).

[38] A. Arhrib, M. Capdequi Peyranere, W. Hollik, and S. Penaranda, Phys. Lett. B 579, 361 (2004).

[39] A. Arhrib, R. Benbrik, J. El Falaki, and W. Hollik, Phys. Lett. B 774, 195 (2017).

[40] T. D. Lee, Phys. Rev. D 8, 1226 (1973).

[41] G. C. Branco, P. M. Ferreira, L. Lavoura, M. N. Rebelo, M. Sher, and J. P. Silva, Phys. Rep. 516, 1 (2012).

[42] S. Davidson and H. E. Haber, Phys. Rev. D 72, 035004 (2005); 72, 099902(E) (2005). 
[43] S. L. Glashow and S. Weinberg, Phys. Rev. D 15, 1958 (1977).

[44] E. A. Paschos, Phys. Rev. D 15, 1966 (1977).

[45] E. Ma, Phys. Rev. D 73, 077301 (2006).

[46] R. Barbieri, L. J. Hall, and V. S. Rychkov, Phys. Rev. D 74, 015007 (2006).

[47] Q.-H. Cao, E. Ma, and G. Rajasekaran, Phys. Rev. D 76, 095011 (2007).

[48] J. F. Gunion and H. E. Haber, Phys. Rev. D 67, 075019 (2003).

[49] V. D. Barger, J. L. Hewett, and R. J. N. Phillips, Phys. Rev. D 41, 3421 (1990).

[50] Y. Grossman, Nucl. Phys. B426, 355 (1994).

[51] N. G. Deshpande and E. Ma, Phys. Rev. D 18, 2574 (1978).

[52] K. A. Olive et al. (Particle Data Group), Chin. Phys. C 38, 090001 (2014).

[53] J. Haller, A. Hoecker, R. Kogler, K. Mönig, T. Peiffer, and J. Stelzer, Eur. Phys. J. C 78, 675 (2018).

[54] F. Mahmoudi, Comput. Phys. Commun. 180, 1579 (2009).

[55] Y. Amhis et al. (HFLAV Collaboration), Eur. Phys. J. C 77, 895 (2017).

[56] Y. Amhis et al. (Heavy Flavor Averaging Group (HFAG)), arXiv:1412.7515.

[57] F. Archilli, in 8th International Workshop on the CKM Unitarity Triangle (CKM 2014) Vienna, Austria, 2014 (2014) [arXiv:1411.4964].

[58] K. Hagiwara, R. Liao, A. D. Martin, D. Nomura, and T. Teubner, J. Phys. G 38, 085003 (2011).

[59] D. Eriksson, J. Rathsman, and O. Stal, Comput. Phys. Commun. 181, 189 (2010).

[60] P. Bechtle, O. Brein, S. Heinemeyer, G. Weiglein, and K. E. Williams, Comput. Phys. Commun. 181, 138 (2010).

[61] P. Bechtle, O. Brein, S. Heinemeyer, G. Weiglein, and K. E. Williams, Comput. Phys. Commun. 182, 2605 (2011).

[62] P. Bechtle, O. Brein, S. Heinemeyer, O. Stal, T. Stefaniak, G. Weiglein, and K. Williams, Proc. Sci., CHARGED2012 (2012) 024 [arXiv:1301.2345].

[63] P. Bechtle, O. Brein, S. Heinemeyer, O. Stal, T. Stefaniak, G. Weiglein, and K. E. Williams, Eur. Phys. J. C 74, 2693 (2014).

[64] P. Bechtle, S. Heinemeyer, O. Stal, T. Stefaniak, and G. Weiglein, Eur. Phys. J. C 75, 421 (2015).

[65] P. Bechtle, S. Heinemeyer, O. Stal, T. Stefaniak, and G. Weiglein, Eur. Phys. J. C 74, 2711 (2014).

[66] O. Stal and T. Stefaniak, Proc. Sci., EPS-HEP2013 (2013) 314 [arXiv:1310.4039].

[67] P. Bechtle, S. Heinemeyer, O. Stal, T. Stefaniak, and G. Weiglein, J. High Energy Phys. 11 (2014) 039.

[68] P. M. Ferreira, R. Santos, M. Sher, and J. P. Silva, Phys. Rev. D 85, 077703 (2012).

[69] J. Bernon, J. F. Gunion, H. E. Haber, Y. Jiang, and S. Kraml, Phys. Rev. D 92, 075004 (2015).

[70] B. Dumont, J. F. Gunion, Y. Jiang, and S. Kraml, Phys. Rev. D 90, 035021 (2014).

[71] K. Cheung, J. S. Lee, and P.-Y. Tseng, J. High Energy Phys. 01 (2014) 085.

[72] O. Eberhardt, U. Nierste, and M. Wiebusch, J. High Energy Phys. 07 (2013) 118.
[73] B. Coleppa, F. Kling, and S. Su, J. High Energy Phys. 01 (2014) 161.

[74] A. Arhrib, R. Benbrik, C.-H. Chen, M. Gomez-Bock, and S. Semlali, Eur. Phys. J. C 76, 328 (2016).

[75] C.-W. Chiang and K. Yagyu, J. High Energy Phys. 07 (2013) 160.

[76] J. Bernon, J. F. Gunion, H. E. Haber, Y. Jiang, and S. Kraml, Phys. Rev. D 93, 035027 (2016).

[77] P. M. Ferreira, R. Santos, M. Sher, and J. P. Silva, Phys. Rev. D 85, 035020 (2012).

[78] M. Misiak and M. Steinhauser, Eur. Phys. J. C 77, 201 (2017).

[79] M. Misiak et al., Phys. Rev. Lett. 114, 221801 (2015).

[80] T. Enomoto and R. Watanabe, J. High Energy Phys. 05 (2016) 002.

[81] A. Arhrib, R. Benbrik, and S. Moretti, Eur. Phys. J. C 77, 621 (2017).

[82] G. Aad et al. (ATLAS Collaboration), J. High Energy Phys. 03 (2015) 088.

[83] V. Khachatryan et al. (CMS Collaboration), J. High Energy Phys. 11 (2015) 018.

[84] V. Khachatryan et al. (CMS Collaboration), J. High Energy Phys. 12 (2015) 178.

[85] G. Aad et al. (ATLAS Collaboration), Eur. Phys. J. C 73, 2465 (2013).

[86] G. Abbiendi et al. (ALEPH, DELPHI, L3, OPAL, and LEP Collaborations), Eur. Phys. J. C 73, 2463 (2013).

[87] A. G. Akeroyd et al., Eur. Phys. J. C 77, 276 (2017).

[88] W. Kilian, M. Kramer, and P. Zerwas, Phys. Lett. B 373, 135 (1996).

[89] M. Carena et al., in CERN Workshop on LEP2 Physics (Followed by 2nd meeting, 15-16 Jun 1995 and 3rd meeting 2-3 Nov 1995) (1996), pp. 351-462 [arXiv:hep$\mathrm{ph} / 9602250]$.

[90] A. Djouadi, Phys. Rep. 459, 1 (2008).

[91] A. Djouadi, J. Kalinowski, and P. M. Zerwas, Z. Phys. C 57, 569 (1993).

[92] M. Bohm, H. Spiesberger, and W. Hollik, Fortschr. Phys. 34, 687 (1986).

[93] W. F. L. Hollik, Fortschr. Phys. 38, 165 (1990).

[94] A. Denner, Fortschr. Phys. 41, 307 (1993).

[95] M. Tanabashi et al. (Particle Data Group), Phys. Rev. D 98, 030001 (2018).

[96] Q.-F. Sun, F. Feng, Y. Jia, and W.-L. Sang, Phys. Rev. D 96, 051301 (2017).

[97] S. Kanemura, Y. Okada, E. Senaha, and C. P. Yuan, Phys. Rev. D 70, 115002 (2004).

[98] S. Kanemura, M. Kikuchi, and K. Yagyu, Nucl. Phys. B896, 80 (2015).

[99] S. Kanemura, M. Kikuchi, K. Sakurai, and K. Yagyu, Phys. Rev. D 96, 035014 (2017).

[100] A. Freitas and D. Stockinger, Phys. Rev. D 66, 095014 (2002).

[101] A. Denner, L. Jenniches, J.-N. Lang, and C. Sturm, J. High Energy Phys. 09 (2016) 115.

[102] A. Denner, J.-N. Lang, and S. Uccirati, J. High Energy Phys. 07 (2017) 087.

[103] A. Denner, S. Dittmaier, and J.-N. Lang, J. High Energy Phys. 11 (2018) 104. 
[104] M. Krause, R. Lorenz, M. Muhlleitner, R. Santos, and H. Ziesche, J. High Energy Phys. 09 (2016) 143.

[105] M. Krause, M. Muhlleitner, R. Santos, and H. Ziesche, Phys. Rev. D 95, 075019 (2017).

[106] D. Binosi and J. Papavassiliou, Phys. Rep. 479, 1 (2009).

[107] L. Altenkamp, S. Dittmaier, and H. Rzehak, J. High Energy Phys. 09 (2017) 134.

[108] L. Altenkamp, S. Dittmaier, and H. Rzehak, J. High Energy Phys. 03 (2018) 110.

[109] M. Krause, M. Muhlleitner, and M. Spira, Comput. Phys. Commun. 246, 106852 (2020).

[110] J.-X. Wang, Nucl. Instrum. Methods Phys. Res., Sect. A 534, 241 (2004).

[111] W. Beenakker, S. C. van der Marck, and W. Hollik, Nucl. Phys. B365, 24 (1991).

[112] Y. Gong, Z. Li, X. Xu, L. L. Yang, and X. Zhao, Phys. Rev. D 95, 093003 (2017).

[113] W. Kilian, T. Ohl, and J. Reuter, Eur. Phys. J. C 71, 1742 (2011).

[114] C. Chen, Z. Cui, G. Li, Q. Li, M. Ruan, L. Wang, and Q.-s. Yan, J. Phys. G 45, 015004 (2018).

[115] J. Alwall, R. Frederix, S. Frixione, V. Hirschi, F. Maltoni, O. Mattelaer, H. S. Shao, T. Stelzer, P. Torrielli, and M. Zaro, J. High Energy Phys. 07 (2014) 079.

[116] S. Kanemura, M. Kikuchi, K. Mawatari, K. Sakurai, and K. Yagyu, Comput. Phys. Commun. 257, 107512 (2020).

[117] M. Krause and M. Muhlleitner, J. High Energy Phys. 04 (2020) 083.

[118] D. de Florian et al. (LHC Higgs Cross Section Working Group), 2/2017, (2016).

[119] S. Kanemura, M. Kikuchi, K. Mawatari, K. Sakurai, and K. Yagyu, Phys. Lett. B 783, 140 (2018).

[120] S. Dawson et al., in Proceedings, 2013 Community Summer Study on the Future of U.S. Particle Physics: Snowmass on the Mississippi (CSS2013): Minneapolis, MN, USA, 2013 (2013) [arXiv:1310.8361].

[121] D. Zeppenfeld, R. Kinnunen, A. Nikitenko, and E. RichterWas, Phys. Rev. D 62, 013009 (2000).
[122] F. Gianotti and M. Pepe-Altarelli, Nucl. Phys. B, Proc. Suppl. 89, 177 (2000).

[123] P. Bambade et al., Reports No. DESY-19-037, No. FERMILAB-FN-1067-PPD, No. IFIC/19-10, IRFU-19-10, No. JLAB-PHY-19-2854, No. KEK Preprint 2018-92, No. LAL/RT 19-001, No. PNNL-SA-142168, No. SLACPUB-17412, 2019, arXiv:1903.01629.

[124] X. Mo, G. Li, M.-Q. Ruan, and X.-C. Lou, Chin. Phys. C 40, 033001 (2016).

[125] E. A. Kuraev and V. S. Fadin, Yad. Fiz. 41, 733 (1985) [Sov. J. Nucl. Phys. 41, 466 (1985)], https://inspirehep.net/ literature/217313.

[126] F. A. Berends and R. Kleiss, Nucl. Phys. B260, 32 (1985).

[127] S. Jadach, M. Skrzypek, and B. Pietrzyk, Phys. Lett. B 456, 77 (1999).

[128] V. N. Gribov and L. N. Lipatov, Yad. Fiz. 15, 1218 (1972) [Sov. J. Nucl. Phys. 15, 675 (1972)], https://inspirehep.net/ literature/73450.

[129] M. Skrzypek and S. Jadach, Z. Phys. C 49, 577 (1991).

[130] E. Barberio and Z. Was, Comput. Phys. Commun. 79, 291 (1994).

[131] S. Jadach, B. F. L. Ward, and Z. Was, Comput. Phys. Commun. 130, 260 (2000).

[132] M. Skrzypek, S. Jadach, W. Placzek, and Z. Was, Comput. Phys. Commun. 94, 216 (1996).

[133] S. Jadach, W. Placzek, M. Skrzypek, B. F. L. Ward, and Z. Was, Comput. Phys. Commun. 140, 475 (2001).

[134] R. Barate et al. (ALEPH Collaboration), Eur. Phys. J. C 14, 1 (2000).

[135] S. Schael et al. (ALEPH, DELPHI, L3, OPAL, and LEP Electroweak Collaborations), Phys. Rep. 532, 119 (2013).

[136] S. Komamiya, Phys. Rev. D 38, 2158 (1988).

[137] A. Arhrib and G. Moultaka, Nucl. Phys. B558, 3 (1999).

[138] G. 't Hooft and M. J. G. Veltman, Nucl. Phys. B153, 365 (1979). 